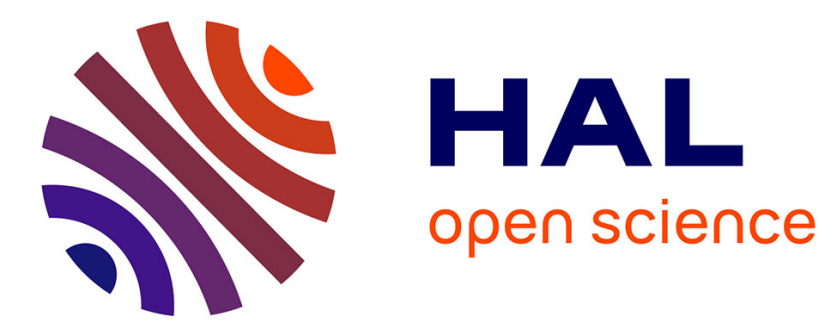

\title{
Hyper-Stable Social Welfare Functions
}

Jean Lainé, Ali Ihsan Ozkes, Remzi Sanver

\section{To cite this version:}

Jean Lainé, Ali Ihsan Ozkes, Remzi Sanver. Hyper-Stable Social Welfare Functions. 2014. hal$00871312 \mathrm{v} 2$

\section{HAL Id: hal-00871312 \\ https://hal.science/hal-00871312v2}

Preprint submitted on 3 Mar 2014

HAL is a multi-disciplinary open access archive for the deposit and dissemination of scientific research documents, whether they are published or not. The documents may come from teaching and research institutions in France or abroad, or from public or private research centers.
L'archive ouverte pluridisciplinaire HAL, est destinée au dépôt et à la diffusion de documents scientifiques de niveau recherche, publiés ou non, émanant des établissements d'enseignement et de recherche français ou étrangers, des laboratoires publics ou privés. 
HYPER-STABLE SOCIAL WELFARE FUNCTIONS

Jean LAINE

Ali Ihsan OZKES

Remzi SANVER

Cahier nº 2014-05 (version révisée 2013-21)

\section{DEPARTEMENT D'ECONOMIE}

Route de Saclay

91128 PALAISEAU CEDEX

(33) 169333033

http://www.economie.polytechnique.edu/

mailto:chantal.poujouly@polytechnique.edu 


\title{
Hyper-Stable Social Welfare Functions*
}

\author{
Jean Lainé ${ }^{\dagger}$
}

Ali Ihsan Ozkes $\ddagger$

M. Remzi Sanver ${ }^{\S}$

February 23, 2014

\begin{abstract}
We introduce a new consistency condition for neutral social welfare functions, called hyperstability. A social welfare function $\alpha$ selects a complete weak order from a profile $P_{N}$ of linear orders over any finite set of alternatives, given $N$ individuals. Each linear order $P$ in $P_{N}$ generates a linear order over orders of alternatives,called hyper-preference, by means of a preference extension. Hence each profile $P_{N}$ generates a hyper-profile $\dot{P}_{N}$. We assume that all preference extensions are separable: the hyper-preference of some order $P$ ranks order $Q$ above order $Q^{\prime}$ if the set of alternative pairs $P$ and $Q$ agree on contains the one $P$ and $Q^{\prime}$ agree on. A special sub-class of separable extensions contains all Kemeny extensions, which build hyper-preferences by using the Kemeny distance criterion. A social welfare function $\alpha$ is hyper-stable (resp. Kemeny-stable) if at any profile $P_{N}$, at least one linearization of $\alpha\left(P_{N}\right)$ is ranked first by $\alpha\left(\dot{P}_{N}\right)$, where $\dot{P}_{N}$ is any separable (resp. Kemeny) hyper-profile induced from $P_{N}$. We show that no scoring rule is hyper-stable, and that no unanimous scoring rule is Kemeny-stable, while there exists a hyper-stable Condorcet social welfare function.
\end{abstract}

Keywords: Hyper-preferences - Kemeny distance - Social Welfare Functions - Stability

\footnotetext{
*We are grateful to the editor and two anonymous referees for helpful comments on an earlier draft.

${ }^{\dagger}$ Corresponding author: Murat Sertel Center for Advanced Economic Studies and Department of Economics, Istanbul Bilgi University Santralistanbul Kampüsü, Eski Silahtarağa Elektrik Santralı, Kazım Karabekir Cad. No: 2/13, Building E2, 34060 Eyüp Istanbul, Turkey. jean. laine@bilgi .edu.tr

$\ddagger$ Murat Sertel Center for Advanced Economic Studies and Department of Economics, Istanbul Bilgi University and Department of Economics, École Polytechnique. ali-ihsan. ozkes@polytechnique.edu

\$Murat Sertel Center for Advanced Economic Studies and Department of Economics, Istanbul Bilgi University. remzi.sanver@bilgi.edu.tr
} 


\section{Introduction}

Many collective choice situations involve orderings of a finite set of $m$ alternatives as resolute outcomes. Natural examples are choosing a social preference or a priority order over decisions, ranking candidates in sport or arts competitions (e.g. the Eurovision song contest) or assigning tasks to individuals. In the latter example, there are $m$ positions to be filled by $m$ individuals, each being assigned a specific position. Given the natural ranking $1>\ldots>m$ of the positions, a social outcome is an or$\operatorname{der} f(1) \succ \ldots \succ f(m)$ over individuals obtained by means of a bijection $f$ from the set of positions to the set of individuals.

The classical framework of social choice theory calls for individuals to report their preferences over social outcomes. When social outcomes are linear orders, preferences over outcomes are orders of orders, or hyper-preferences. However, reporting full preferences faces a problem of practical implementation: in the no-indifference case, individuals have to rank $m$ ! outcomes. More generally, when outcomes are complex combinations of basic alternatives, likewise orderings or subsets, choosing from full preference profiles is hardly achievable in practice. This suggests to design procedures based on partial information about individual preferences. ${ }^{1}$ An immediate option is asking each of the individuals to report only one order. Formally, this procedure reduces to using a Social Welfare Function (SWF) $\alpha$, which maps every profile of linear orders to a weak order of alternatives, completed with a tie-breaking rule.

It follows that some normative properties of SWFs cannot be investigated without retaining assumptions on how individual orders over alternatives are extended to underlying hyper-preferences. A typical example is given by strategy-proofness, which can be defined only conditional to the way orders over alternatives are extended to hyper-preferences. Bossert and Storcken (1992) prove impossibility results for hyper-preferences generated by the criterion of Kemeny distance: given an order $P$ over alternatives, the hyper-preference from $P$ ranks an order $Q$ above another order $Q^{\prime}$ if the Kemeny distance between $P$ and $Q$ is strictly lower than the one between $P$ and $Q^{\prime}{ }^{2}$ Bossert and Sprumont (2012) investigate strategy-proofness for hyper-preferences based on the following betweenness criterion: the hyper-preference from $P$ ranks $Q$ above $Q^{\prime}$ if the set of alternative pairs $P$ and $Q$ agree on contains the set of pairs $P$ and $Q^{\prime}$ agree on. ${ }^{3}$ Another property requiring extending orders to hyperpreferences is the Pareto property, which states that an SWF (with a tie-breaking rule) chooses at any profile over alternatives a linear order that is not unanimously less preferred than another order.

In this paper we introduce a new property for neutral SWFs, called hyper-stability, which also implies linking orders over alternatives to hyper-preferences. Hyper-stability is a consistency property relating two levels of choice, the one from profiles of orders over alternatives, called basic profiles, and the one from hyper-preference profiles, or hyper-profiles. Loosely speaking, an SWF is hyper-stable if its outcomes at any basic profile is top-ranked at the corresponding hyper-profile. More precisely, consider an SWF $\alpha$ defined for any finite number of alternatives. Hence, $\alpha$ provides a weak order at any basic profile over $m$ alternatives as well as from any basic profile over $m$ ! alternatives. Furthermore, suppose that $\alpha$ is neutral, meaning that its outcomes are non-sensitive to the labeling of alternatives. Thus, profiles over $m$ ! alternatives can be also interpreted as hyper-preference profiles

\footnotetext{
${ }^{1}$ This is what prevails in the Eurovision song contest, where ballots are based on a partial scoring method.

${ }^{2}$ The Kemeny distance between two linear orders is the number of pairs of alternatives which they disagree on.

${ }^{3}$ See Duddy et al. (2010) for an analysis of strategy-proof SWFs based on ordinally fuzzy preferences.
} 
over orders of $m$ alternatives, or in short hyper-profiles. While a basic profile clearly entails a huge loss of information about preferences over outcomes, there may nonetheless exist, in the spirit of revealed-preference theory, a class of underlying hyper-profiles (over $m$ ! orders) compatible with the basic profile at which $\alpha$ ranks at top at least one linearization of the weak order chosen from the basic profile. If this happens at every possible reduced profile, we say that $\alpha$ is hyper-stable.

As for strategy-proofness, a key-issue for hyper-stability is what is meant by a class of hyperprofiles compatible with a basic profile. We assume here that compatibility holds when hyperpreferences are generated from orders over alternatives in accordance with the betweenness criterion. Clearly, this criterion allows to compare only a small number of orders, therefore a basic profile generates a large class of compatible hyper-profiles. Nonetheless, we prove the existence of a unanimous and hyper-stable Condorcet SWF. ${ }^{4}$ However, many well-known Condorcet SWFs are not hyper-stable.

We also pay attention to the sub-class of hyper-profiles built by means of the Kemeny distance criterion. Hyper-stability relative to this sub-class is called Kemeny-stability. We show that no scoring rule is Kemeny-stable, hence hyper-stable. Then, our main result is that ranking by scoring is incompatible with hyper-stability, while the Condorcet criterion is not.

To the best of our knowledge, hyper-stability is a new property for SWFs, although related properties appear in several studies of collective choice. The yeast of the present study can be found in Binmore (1975), who considers a stronger notion of hyper-stability, although in a different setting. Suppose that preferences are now weak orders over three alternatives, which are aggregated to a weak order by means of a neutral SWF $\alpha$. Binmore does not comment on hyper-preferences beyond writing "if a rational entity holds a certain preference preordering over a set of alternatives, then that entity must also subscribe to a certain partial preordering of the set of all preorderings" (Binmore, 1975, page 379). Moreover, weak orders are compared according to their respective top-sets. All relevant top-sets in Binmore's analysis contain at most two elements and the criterion works as follows: Given a weak order $R$, sets $\{x\},\{y\}$, and $\{x, y\}$ are ranked in the order $\{x\},\{x, y\},\{y\}$ if and only if $x R y$. Given the 13 possible weak orders over 3 alternatives, this criterion suffices to find a family $\mathcal{T}$ of triples of weak orders on which basic preferences generate a hyper-profile. ${ }^{5}$ Since $\alpha$ is neutral, it can be applied to each of these hyper-profiles, leading to a weak order $R_{T}$ over each triple $T$ in $\mathcal{T}$. Furthermore, the weak order chosen from the basic profile also induces a weak order $\widetilde{R}_{T}$ over each triple $T$ in $\mathcal{T}$. Binmore shows that $R_{T}$ and $\widetilde{R}_{T}$ coincide for all $T$ in $\mathcal{T}$ if and only if $\alpha$ is dictatorial, anti-dictatorial or constant. There are three main differences between Binmore's approach and the present one. First, basic preferences and hyper-preferences are weak orders in Binmore's study, while we assume both are linear orders. Second, Binmore's setting defines SWFs for three alternatives only. Using neutrality together with a way to generate hyper-preferences, this allows to choose from hyper-profiles over triples of orders. In contrast, our setting involves a variable

\footnotetext{
${ }^{4}$ An alternative is a Condorcet winner at some basic profile if it defeats all other alternatives according to the majority rule. An SWF is Condorcet if it uniquely ranks first a Condorcet winner whenever it exists.

${ }^{5}$ To see why, label alternatives as $x, y$ and $z$, and consider the following weak orders $R_{1}, R_{2}$ and $R_{3}$ (with respective a-symmetric parts $P_{1}, P_{2}$ and $P_{3}$ ) defined by $z P_{1} y P_{1} x, y P_{2} z P_{2} x$ and $y R_{3} z P_{3} x$. Denote by $\succsim_{1}, \succsim_{2}$ and $\succsim_{3}$ the respective hyper-preferences induced on $\left\{R_{1}, R_{2}, R_{3}\right\}$ by $R_{1}, R_{2}$ and $R_{3}$. Then one gets $R_{1} \succ_{1} R_{3} \succ_{1} R_{2}, R_{2} \succ_{2} R_{3} \succ_{2} R_{1}$ and $R_{1} \sim_{3} R_{2} \sim_{3} R_{3}$. It is easily seen that for each of the 13 possible weak orders, $R_{1}, R_{2}$ and $R_{3}$ are ranked as in $\succsim_{1}$, or $\succsim_{2}$ or $\succsim_{3}$. Hence, any basic profile generates an hyper-profile over triple $\left\{R_{1}, R_{2}, R_{3}\right\}$.
} 
number of alternatives, and defines hyper-preferences as linear orders over all orders. Again, using neutrality together with a way to generate hyper-preferences, this allows to have a well-defined outcome at profiles over $m$ alternatives and at hyper-profiles over $m$ ! orders. Third, our definition of hyper-stability is clearly less demanding than Binmore's one, since it only requires that some social order chosen from basic profiles is top-ranked from hyper-profiles, imposing nothing about how this social order itself generates a social hyper-preference.

Another study related to hyper-stability can be found in Laffond and Lainé (2000), although the property is not explicitly stated there. Using the same framework as the present one, Laffond and Lainé characterize the class of (neutral and independent) hyper-preferences such that whenever the majority tournament at a basic profile is transitive, it is a Condorcet winner of any corresponding hyper-profile. This characterization result can be restated as follows in terms of hyper-stability. Call strongly Condorcet a SWF which gives as outcome the majority tournament whenever it is transitive. Then every strongly Condorcet SWF is hyper-stable relative to some class of hyper-preferences. .

Hyper-stability also appear, at least in watermark, in the literature of moral judgments. ${ }^{6}$ Sen (1974) argues that morality requires to formulate judgments among preferences while rationality does not, and suggests using moral views, defined as hyper-preferences, as a way out of the Paretian liberal paradox à la Sen (1970). ${ }^{7}$ If one accepts basic profiles as expressions of rationality (individuals reporting their first-best outcome) and hyper-profiles as expressions of moral judgments, hyper-stability can be interpreted as a property of moral consistency: choices made from rational preferences does not conflict with the one made from moral judgments.

Furthermore, hyper-stability can be related to a property of self-selectivity for SWFs. Self-selectivity is defined for a social choice function (SCF) by Koray (2000). ${ }^{8}$ Roughly speaking, an SCF is selfselective if it chooses itself against any finite number of other social choice functions. Self-selectivity thus involves two levels of choice: choices from profiles over alternatives, and choices from profiles over choice functions. These two levels are connected by means of a consequentialist principle, which states that individuals preferring alternative $x$ to alternative $y$ will rank any function choosing $x$ above any function choosing $y$. Koray (2000) shows that a neutral and unanimous SCF is selfselective if and only if it is dictatorial. While consequentialism allows for a canonical extension of preferences over alternatives to preferences over SCFs, this is no longer the case for SWFs. Nonetheless, self-selectivity for SWFs can be defined conditional to the definition of hyper-preferences. An individual with preference $P$ in some basic profile $P_{N}$ will prefer SWF $\alpha_{1}$ to SWF $\alpha_{2}$ if $\alpha_{1}\left(P_{N}\right)$ is "closer" to $P$ than $\alpha_{2}\left(P_{N}\right)$, where closer can be in terms of the Kemeny or any other distance. More generally, once defined how $P$ generates a hyper-preference $\dot{P}$, two SWFs being compared according to the way $\dot{P}$ ranks their respective outcomes. Hence the consequentialist principle applies, but conditional to the way basic preferences are extended to hyper-preferences. We say that a SWF is $S W$ self-selective for some preference extension if, at any basic profile it ranks itself first when compared to any finite set of SWFs. We show below that hyper-stability is a necessary condition for SW self-selectivity.

\footnotetext{
${ }^{6}$ One can think of hyper-preferences also as preferences of individuals over others in the society.

${ }^{7}$ See Igersheim (2007). The reader may refer to Jeffrey (1974), McPherson (1982), and Sen (1977) for further discussion on the more general concept of a meta-preference.

${ }^{8} \mathrm{~A}$ social choice function picks one alternative at every profile of preferences over alternatives. For further studies of self-selectivity, see Koray and Unel (2003) and Koray and Slinko (2008).
} 
The rest of the paper is organized as follows. Part 2 formally defines hyper-stability, and investigates its relation to self-selectivity. Hyper-stability of scoring rules is studied in Part 3. In particular, we provide examples showing that neither the Borda rule, nor the plurality and anti-plurality rules are Kemeny-stable, hence hyper-stable. Moreover, we show that no unanimous scoring rule is Kemeny-stable, and that no scoring rule is hyper-stable. Condorcet SWFs are considered in Part 4. We show that the Slater SWF, the Kemeny rule, and the Copeland SWF are not hyper-stable, whereas the transitive closure of the majority relation over alternatives is hyper-stable. The paper ends up with comments about alternative concepts of hyper-stability, together with open questions. Finally, all proofs are postponed to an appendix.

\section{Hyper-stability}

\subsection{Notations and definitions}

Let $\mathbb{N}$ be the set of non-zero natural numbers. We consider societies with variable numbers of individuals and of alternatives. Hence, $\mathbb{N}$ stands for the sets of potential alternatives and individuals, and each actual society involves finitely many individuals confronting finitely many alternatives. Given $m \in \mathbb{N}$, we define $A_{m}=\{1, \ldots, m\}$ as a set of $m$ social alternatives. The set of linear (resp. weak) orders over $A_{m}$ is denoted by $\mathcal{L}\left(A_{m}\right)$ (resp. $\mathcal{R}\left(A_{m}\right)$ ). An order $P \in \mathcal{L}\left(A_{m}\right)$ is a linear extension of $R \in \mathcal{R}\left(A_{m}\right)$ if for any $a, b \in A_{m}, a P b \Rightarrow a R b$. The set of all linear extensions of $R \in \mathcal{R}\left(A_{m}\right)$ is denoted by $\Delta(R)$. Given a set $N$ of $n$ individuals, a weak profile is an element $R_{N}$ of $\mathcal{R}\left(A_{m}\right)^{n}$, and a profile is an element $P_{N}$ of $\mathcal{L}\left(A_{m}\right)^{n}$. The set of all linearizations of the weak profile $R_{N}$ is $\Delta\left(R_{N}\right)=\times_{i \in N}\left(\Delta\left(R_{i}\right)\right)$.

A function $\alpha$ from $\cup_{m, n \in \mathbb{N}} \mathcal{L}\left(A_{m}\right)^{n}$ to $\cup_{m \in \mathbb{N}} \mathcal{R}\left(A_{m}\right)$ is a social welfare function (SWF) if, for all $n, m \in$ $\mathbb{N}$ and all $P_{N} \in \mathcal{L}\left(A_{m}\right)^{n}, \alpha\left(P_{N}\right) \in \mathcal{R}\left(A_{m}\right)$. Moreover, a SWF $\alpha$ is neutral if for all $n, m \in \mathbb{N}$ and all $P_{N}=\left(P_{1}, \ldots, P_{n}\right) \in \mathcal{L}\left(A_{m}\right)^{n}$, for all $a, b \in A_{m}, a \alpha\left(P_{N}\right) b$ if and only if $\gamma(a) \alpha\left(P_{N}^{\gamma}\right) \gamma(b)$. Note that since $\alpha$ are defined for any number of alternatives, neutrality ensures that the precise labeling of alternatives does not matter. In particular, $\alpha$ is defined for profiles over $m$ ! alternatives, which can be either basic alternatives or linear orders over $m$ basic alternatives. Furthermore, a SWF $\alpha$ is unanimous if, for any $m, n \in \mathbb{N}$, for any profile $P_{N} \in \mathcal{L}\left(A_{m}\right)^{n}$, for any two alternatives $a, b \in A_{m}$, $\left[a P_{i} b\right.$ for all $\left.i=1, \ldots, n\right]$ implies that $\left[a \alpha\left(P_{N}\right) b\right.$ and $\left.\neg\left(b \alpha\left(P_{N}\right) a\right)\right]$. Given a SWF $\alpha$, the $\alpha$-induced correspondence $f_{\alpha}: \cup_{n, m \in \mathbb{N}} \mathcal{L}\left(A_{m}\right)^{n} \rightarrow 2^{A_{m}} \backslash \varnothing$ is defined by: $\forall n, m \in \mathbb{N}, \forall P_{N} \in \mathcal{L}\left(A_{m}\right)^{n}, \forall a \in A_{m}$, $a \in f_{\alpha}\left(P_{N}\right) \Longleftrightarrow a \alpha\left(P_{N}\right) b$ for all $b \in A_{m}$. Hence, the $\alpha$-induced correspondence selects at each profile $P_{N}$ the top-set for $\alpha\left(P_{N}\right)$.

\subsection{Preference extensions}

We turn now to the notion of hyper-preference. A preference extension is a function $e: \cup_{m} \in \mathbb{N} \mathcal{L}\left(A_{m}\right) \rightarrow$ $\cup_{m \in \mathbb{N}} \mathcal{L}\left(\mathcal{L}\left(A_{m}\right)\right)$ such that for all $m \in \mathbb{N}$ and all $P \in \mathcal{L}\left(A_{m}\right), e(P) \in \mathcal{L}\left(\mathcal{L}\left(A_{m}\right)\right)$. Hence, a preference extension maps each linear order over $m$ alternatives to a linear order over all linear orders over alternatives. An element of $\mathcal{L}\left(\mathcal{L}\left(A_{m}\right)\right)$ is called hyper-preference. An extension domain is a proper subset $\mathcal{E}$ of the set of all preference extensions. Given a profile $P_{N}=\left(P_{1}, \ldots, P_{n}\right) \in \mathcal{L}\left(A_{m}\right)^{n}$ together 
with a $n$-tuple $E=\left(e_{1}, \ldots, e_{n}\right) \in \mathcal{E}^{n}$, an hyper-profile of $P_{N}$ is the element $P_{N}^{E}=\left(e_{1}\left(P_{1}\right), \ldots, e_{n}\left(P_{n}\right)\right)$.

Given $P, Q \in \mathcal{L}\left(A_{m}\right)$, we define the set $A(P, Q)=\left\{(a, b) \in A_{m} \times A_{m}: a P b\right.$ and $\left.a Q b\right\}$, which contains all alternative pairs $P$ and $Q$ agree on. We focus on the specific class of separable preference extensions.

Definition 1 A preference extension e is separable if for all $m \in \mathbb{N}$ and all $P, Q, Q^{\prime} \in \mathcal{L}\left(A_{m}\right), A(P, Q) \supset$ $A\left(P, Q^{\prime}\right)$ implies $Q e(P) Q^{\prime}$.

We denote by $\mathcal{S}$ the domain of separable preference extensions.

Given $P, Q \in \mathcal{L}\left(A_{m}\right)$, the Kemeny distance between $P$ and $Q$ is defined by $d_{K}(P, Q)=$ $\mid\left\{(a, b) \in A_{m} \times A_{m}: a P b\right.$ and $\left.b Q a\right\} \mid$, that is the number of pairs of alternatives $P$ and $Q$ disagree on.

Definition 2 A preference extension $e$ is Kemeny if for all $m \in \mathbb{N}$ and all $P, Q, Q^{\prime} \in \mathcal{L}\left(A_{m}\right), d_{K}(P, Q)<$ $d_{K}\left(P, Q^{\prime}\right)$ only if $Q e(P) Q^{\prime}$.

We denote by $\mathcal{K}$ the domain of Kemeny preference extensions. Pick up any $P \in \mathcal{L}\left(A_{m}\right)$. Using Kemeny distance allows to induce from $P$ the element $\succsim_{P} \in \mathcal{R}\left(\mathcal{L}\left(A_{m}\right)\right)$ defined by: $\forall Q, Q^{\prime} \in \mathcal{L}\left(A_{m}\right)$, $Q \succsim_{P} Q^{\prime}$ iff $d_{K}(P, Q) \leq d_{K}\left(P, Q^{\prime}\right)$, and $Q \succ_{P} Q^{\prime}$ iff $d_{K}(P, Q)<d_{K}\left(P, Q^{\prime}\right)$. In words, the weak order $\succsim_{P}$ induced by $P$ ranks orders according to their respective distances to $P$. Given profile $P_{N}=$ $\left(P_{1}, \ldots, P_{n}\right) \in \mathcal{L}\left(A_{m}\right)^{n}$, the Kemeny weak profile for $P_{N}$ is defined by $P_{N}^{K}=\left(\succsim_{P_{1}}, \ldots, \succsim_{P_{n}}\right)$. Thus, a preference extension $e$ is Kemeny if for all $m \in \mathbb{N}$ and all $P \in \mathcal{L}\left(A_{m}\right), e$ is a linear extension of $\succsim_{p}$. We call Kemeny hyper-profile any linearization of $P_{N}^{K}$. Clearly, every Kemeny extension is separable, and thus $\mathcal{K} \subset \mathcal{S}$.

The Kemeny distance criterion can be criticized by arguing that when comparing two orders, inversions in the lower tail of the ranking are less important that inversions in the upper tail. If three candidates $a, b, c$ are to be ranked as gold, silver and bronze medal, and if your own ranking is $a P b P c$, then you should prefer order $a Q c Q b$ to order $b Q^{\prime} a Q^{\prime} c$, since reversing order for gold and silver seems appears as a more significant deviation than reversing order for silver and bronze. This calls for breaking symmetry by using weighted Kemeny distance (equivalently, this calls for some specific way to break ties in the Kemeny weak profiles). Note however that such a critic no longer holds if agendas are interpreted as task assignments. Indeed, suppose that $a Q c Q b$ stands for assigning task 1 to individual $a$ task 2 to $c$ and task 3 to $b$, a similar meaning being given to $Q^{\prime}$. Provided that all task are given the same importance, $Q$ and $Q^{\prime}$ involve only one mismatch from the viewpoint $P$, and nothing suggests why $Q$ should be preferred to $Q^{\prime}$.

The following example illustrates the construction of Kemeny hyper-profiles. Consider the following profile $P_{N}=\left(P_{1}, P_{2}, P_{3}\right)$ over 3 alternatives $a, b, c$ :

$$
P_{N}=\left(\begin{array}{lll}
P_{1} & P_{2} & P_{3} \\
\hline a & c & c \\
b & b & a \\
c & a & b
\end{array}\right)
$$

The Kemeny weak profile $P_{N}^{K}$ of $P_{N}$ is defined by 


$$
P_{N}^{K}=\left(\begin{array}{ccc}
\succsim P_{1} & \succsim_{P_{2}} & \succsim_{P_{3}} \\
\hline a b c & c b a & c a b \\
a c b, b a c & b c a, c a b & c b a, a c b \\
b c a, c a b & a c b, b a c & a b c, b c a \\
c b a & a b c & b a c
\end{array}\right)
$$

where $x y z$ stands for the linear order $x P y P z$, and where two orders belonging to the same row and column are indifferent. A Kemeny hyper-profile for $P_{N}$ is any element $\dot{P}_{N}$ of $\Delta\left(P_{N}^{K}\right)$. For instance,

$$
\dot{P}_{N}=\left(\begin{array}{ccc}
\dot{P}_{1} & \dot{P}_{2} & \dot{P}_{3} \\
\hline a b c & c b a & c a b \\
b a c & c a b & c b a \\
a c b & b c a & a c b \\
b c a & b a c & a b c \\
c a b & a c b & b c a \\
c b a & a b c & b a c
\end{array}\right)
$$

Contrarily to the Kemeny distance criterion, separability does not automatically induces a weak order over orders. For instance, $e\left(P_{1}\right) \in \mathcal{S}$ only if the following conditions holds: $(1) e\left(P_{1}\right)$ uniquely ranks $P_{1}$ first and its inverse $c b a$ last, (2) $a c b$ is ranked above $c a b$, and (3) $b a c$ is ranked above $b c a$. The reader will easily check that hyper-profile $\widetilde{P}_{N}$ below is built from a vector of separable preference extensions which are not Kemeny.

$$
\widetilde{P}_{N}=\left(\begin{array}{ccc}
\widetilde{P}_{1} & \widetilde{P}_{2} & \widetilde{P}_{3} \\
\hline a b c & c b a & c a b \\
b a c & c a b & c b a \\
b c a & b a c & a c b \\
a c b & b c a & a b c \\
c a b & a c b & b c a \\
c b a & a b c & b a c
\end{array}\right)
$$

\subsection{Hyper-stability: definition}

We are now ready to formally define hyper-stability:

Definition 3 Given $n \in \mathbb{N}$, a neutral social welfare function $\alpha$ is hyper-stable for the domain $\mathcal{E}$ of preference extensions if for all $m, n \in \mathbb{N}$, for all $P_{N} \in \mathcal{L}\left(A_{m}\right)^{n}$, for all $E=\left(e_{1}, \ldots, e_{n}\right) \in \mathcal{E}^{n}$, we have $\Delta\left(\alpha\left(P_{N}\right)\right) \cap$ $f_{\alpha}\left(P_{N}^{E}\right) \neq \varnothing$. Moreover, $\alpha$ is Kemeny-stable if it is hyper-stable for $\mathcal{K}$.

A neutral SWF $\alpha$ is hyper-stable for domain $E$ if at every finite profile $P_{N}$ of linear orders over $m$ alternatives, at least one linear extension of the weak order $\alpha\left(P_{N}\right)$ is ranked first by $\alpha$ when applied to any hyper-profile $P_{N}^{E}$ induced from $P_{N}$ by a vector of preference extensions in $\mathcal{E}$.

Figure 1 below illustrates hyper-stability.

A society with size $n$ has to rank $m$ alternatives, and has agreed on some SWF $\alpha$ as voting rule. Hence, individual ballots are linear orders of alternatives (profile $P_{N}$ ), and ballots are aggregated by 


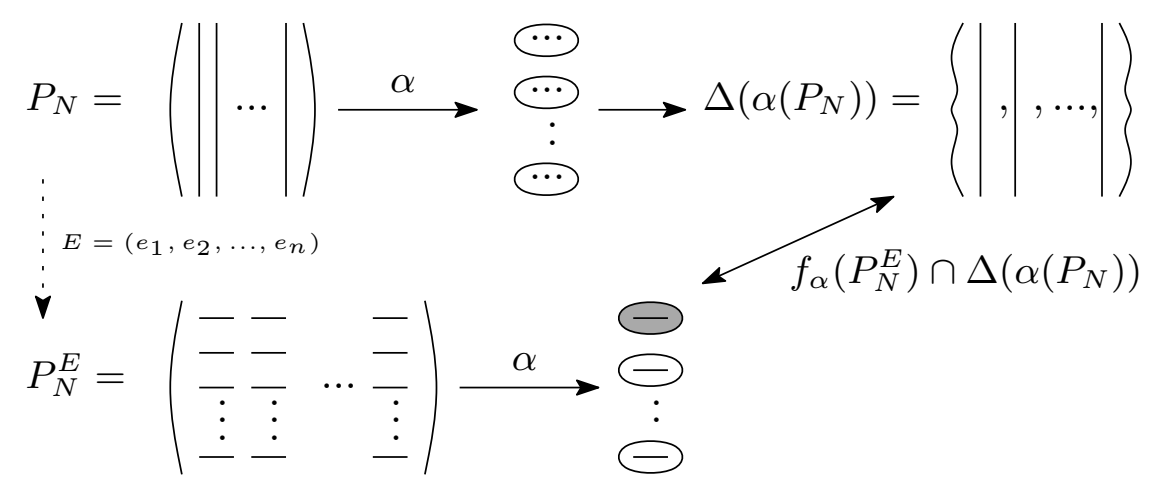

Figure 1: Hyper-stability.

means of $\alpha$ to a weak order $\alpha\left(P_{N}\right)$ of alternatives. Since $\alpha\left(P_{N}\right)$ may involve ties, and since resolute outcomes are linear orders, the final choice results from the use of some tie-breaking rule. The set $\Delta\left(\alpha\left(P_{N}\right)\right)$ contains all possible outcomes obtained by a tie-breaking rule. Ballots provide little information about preferences over outcomes. We assume that "preferences behind ballots" are induced from ballots by some $n$-tuple $E=\left(e_{1}, \ldots, e_{n}\right)$ of preference extensions. Therefore, the set of ballots $P_{N}$ together with $E$ generates a profile $P_{N}^{E}$ over orders, or hyper-profile. Since $\alpha$ is neutral and defined for any number of alternatives, it can be applied to $P_{N^{\prime}}^{E}$ leading to a weak order $\alpha\left(P_{N}^{E}\right)$ over outcomes. Hyper-stability prevails for $\left(e_{1}, \ldots, e_{n}\right)$ if at least one possible final outcome from ballots is ranked first by $\alpha$ (or, equivalently, chosen by $f_{\alpha}$ ) at any full preference profile.

\subsection{Hyper-stability and SW self-selectivity}

While the main motivation for studying hyper-stability is that ballots can hardly indicate full preferences over outcomes, another one stems from its close relationship with self-selectivity. Selfselectivity is defined by Koray (2000) for SCFs. ${ }^{9}$ Suppose that the society has to choose one alternative among finitely many, as well as the SCF itself. Moreover, suppose that given individual preferences over alternatives, individuals compare SCFs by considering only their respective outcomes. According to this consequentialist principle, initial preferences over alternatives naturally extend to preferences over SCFs: consider any finite subset $\mathcal{G}$ of neutral SCFs together with a profile $P_{N}=\left(P_{1}, \ldots, P_{n}\right) \in \mathcal{L}\left(A_{m}\right)^{n}$; define for all $i=1, \ldots, n$ the weak order $R\left(P_{i}\right)$ over $\mathcal{G}$ by: $\forall F, G \in \mathcal{G}, F$ $R^{+}\left(P_{i}\right) G \Leftrightarrow F\left(P_{N}\right) P_{i} G\left(P_{N}\right)$, and $F R^{\sim}\left(P_{i}\right) G \Leftrightarrow F\left(P_{N}\right)=G\left(P_{N}\right)$, where $R^{+}\left(P_{i}\right)$ (resp. $R^{\sim}\left(P_{i}\right)$ ) is the a-symmetric (resp. symmetric) part of $R\left(P_{i}\right)$. It follows that $P_{N}$ induces a dual profile of weak orders $P_{N}^{\mathcal{G}}=\left(R\left(P_{1}\right), \ldots, R\left(P_{n}\right)\right)$ over $\mathcal{G}$. Self-selectivity holds for a SCF $F$ if, at any profile over alternatives, $F$ selects itself some linearization of the dual profile over any finite set of SCFs. Formally, $F$ is selfselective if for all $m, n \in \mathbb{N}$, for all $P_{N} \in \mathcal{L}\left(A_{m}\right)^{n}$, for all finite subsets $\mathcal{G}$ of neutral SCFs with $F \in \mathcal{G}$, there exists a linearization $\widetilde{P}_{N}^{\mathcal{G}}$ of $P_{N}^{\mathcal{G}}$ with $F\left(\widetilde{P}_{N}^{\mathcal{G}}\right)=F$. Koray (2000) proves that, given any fixed size

\footnotetext{
${ }^{9}$ Formally, a function $F: \cup_{m, n \in \mathbb{N}} \mathcal{L}\left(A_{m}\right)^{n} \rightarrow \cup_{m \in \mathbb{N}} A_{m}$ is a social choice function (SCF) if for all $n, m \in \mathbb{N}$ and all $P_{N}$ $\in \mathcal{L}\left(A_{m}\right)^{n}, F\left(P_{N}\right) \in A_{m}$. Furthermore A SCF $F$ is neutral if for all $n, m \in \mathbb{N}$ and all $P_{N}=\left(P_{1}, \ldots, P_{n}\right) \in \mathcal{L}\left(A_{m}\right)^{n}$, for any permutation $\gamma$ of $A_{m}, F\left(P_{N}^{\gamma}\right)=\gamma\left(F\left(P_{N}\right)\right)$, where $P_{N}^{\gamma}=\left(P_{1}^{\gamma}, \ldots, P_{n}^{\gamma}\right) \in \mathcal{L}\left(A_{m}\right)^{n}$ is defined by: $\forall i \in\{1, \ldots, n\}, \forall a, b \in A_{m}$, $a P_{i} b$ if and only if $\gamma(a) P_{i}^{\gamma} \gamma(b)$.
} 
$n$ of the society, a neutral and unanimous SCF is self-selective if and only if it is dictatorial. ${ }^{10}$

Self-selectivity for neutral SWFs is defined along the same lines: at any profile over alternatives, a self-selective SWF ranks itself first among finitely many other SWFs. However, since a SWF provides a weak order, there is no longer a natural duality between preferences over alternatives and preferences over SWFs. In order to make the consequentialist principle meaningful, we need to connect both preference levels by means of a preference extension. It follows that self-selectivity is defined conditional to some domain of preference extensions. This last point is the major difference between the SCF and the SWF settings: choosing preference extensions brings an extra degree of freedom in the analysis, which may allow to escape from Koray's impossibility result.

We formalize self-selectivity for SWFs as follows. A SWF $\alpha$ is called strict if for all $n, m \in \mathbb{N}$ and all $P_{N} \in \mathcal{L}\left(A_{m}\right)^{n}$, one has $\alpha\left(P_{N}\right) \in \mathcal{L}\left(A_{m}\right)$. A linearization of SWF $\alpha$ is a strict SWF $\alpha^{*}$ such that for all $n, m \in \mathbb{N}$, for all $a, b \in A_{m}$ and for all $P_{N} \in \mathcal{L}\left(A_{m}\right)$, one has , $a \alpha^{*}\left(P_{N}\right) b$ only if $a \alpha\left(P_{N}\right) b$. The set of all linearizations of $\alpha$ is denoted by $L(\alpha)$. Pick up a profile $P_{N}=\left(P_{1}, \ldots, P_{n}\right) \in \mathcal{L}\left(A_{m}\right)^{n}$ together with a domain $\mathcal{E}$, and consider any finite subset $\mathcal{A}=\left\{\alpha_{1}, \ldots, \alpha_{K}\right\}$ of neutral SWFs. A strict selection of $\mathcal{A}$ is a subset $\mathcal{A}^{*}=\left\{\alpha_{1}^{*}, \ldots, \alpha_{K}^{*}\right\}$ of linearizations of $\alpha_{1}, \ldots, \alpha_{K}$. For all $1 \leq i \leq n$, define the weak order $\succsim_{P_{i}}^{\mathcal{A}^{*}}$ over $\mathcal{A}^{*}$ by: $\forall 1 \leq k, k^{\prime} \leq K, \alpha_{k}^{*} \succ \mathcal{A}_{P_{i}}^{\mathcal{A}^{*}} \alpha_{k^{\prime}}^{*} \Leftrightarrow \alpha_{k}^{*}\left(P_{N}\right) e_{i}\left(P_{i}\right) \alpha_{k^{\prime}}^{*}\left(P_{N}\right)$, and $\alpha_{k}^{*} \sim_{P_{i}}^{\mathcal{A}^{*}} \alpha_{k^{\prime}}^{*} \Leftrightarrow \alpha_{k}^{*}\left(P_{N}\right)$ $=\alpha_{k^{\prime}}^{*}\left(P_{N}\right)$ for some $\left(e_{1}, \ldots, e_{n}\right) \in \mathcal{E}^{n}$. Thus, as for SCFs, $P_{N}$ together with $E=\left(e_{1}, \ldots, e_{n}\right) \in \mathcal{E}^{n}$ induces a dual profile of weak orders $P_{N}^{E \mathcal{A}^{*}}=\left(\succsim_{P_{1}^{*}}^{\mathcal{A}^{*}}, \ldots, \succsim_{P_{n}}^{\mathcal{A}^{*}}\right)$ over $\mathcal{A}^{*}$.

Definition 4 A neutral SWF $\alpha$ is SW self-selective for the domain of preference extensions $E$ if and only if for all $m, n \in \mathbb{N}$, for all $P_{N} \in \mathcal{L}\left(A_{m}\right)^{n}$, for all finite subsets $\mathcal{A}$ of neutral SWFs that contain $\alpha$, for all strict selection $\mathcal{A}^{*}$ of $\mathcal{A}$, for any $E=\left(e_{1}, \ldots, e_{n}\right) \in \mathcal{E}^{n}$, there exists a linearization $\widetilde{P}_{N}^{E \mathcal{A}^{*}}$ of $P_{N}^{E \mathcal{A}^{*}}$ for which $L(\alpha) \cap \mathcal{A}^{*} \cap f_{\alpha}\left(\widetilde{P}_{N}^{E \mathcal{A}^{*}}\right) \neq \varnothing$.

A neutral SWF $\alpha$ is SW self-selective for domain $\mathcal{E}$ if the following holds: pick up any strict selection $\mathcal{A}^{*}$ of any finite set $\mathcal{A}$ of neutral SWFs including $\alpha$, together with any profile $P_{N}$ over alternatives. Picking up $n$ preference extensions in $\mathcal{E}$ generates from $P_{N}$ a dual profile of weak orders over $\mathcal{A}^{*}$. Then, there exists a linearization of this dual profile at which $\alpha$ ranks first at least some of its linearizations in $\mathcal{A}^{*}$.

Note that, although it offers a natural adaptation of the original concept to SWFs, the formalization of SW self-selectivity sounds complex for two main reasons. First, two different SWFs may have the same outcome at some profile $P_{N}$. Therefore, choosing a domain $\mathcal{E}$ is not enough to provide a dual profile of linear orders over SWFs. Second, two SWFs may produce different weak orders at $P_{N}$ that admit the same linearization. Moreover, note the crucial role played by neutrality, which allows for $\alpha$ to be well-defined for profiles over alternatives and for dual profiles over SWFs.

Proposition 1 below states that hyper-stability is a weaker property than SW self-selectivity.

Proposition 1 A neutral SWF is SW self-selective for domain $\mathcal{E}$ only if it is hyper-stable for $\mathcal{E}$.

\footnotetext{
${ }^{10} \mathrm{~A}$ SCF $F$ is dictatorial if $\exists 1 \leq i \leq n$ such that, for all $P_{N} \in \mathcal{L}\left(A_{m}\right)^{n}, F\left(P_{N}\right)=a \Leftrightarrow a P_{i} b$ for all $b \in A_{m} /\{a\}$. Moreover, $F$ is unanimous if for any $m$, for any $P_{N} \in \mathcal{L}\left(A_{m}\right)^{n}$, for all $a, b \in A_{m},\left[a P_{i} b\right.$ for all $\left.1 \leq i \leq n\right] \Rightarrow b \notin F\left(P_{N}\right)$.
} 


\section{Scoring rules}

We first study hyper-stability of scoring rules. Given a number $m$ of alternatives, a score vector is an element $S^{m}=\left(s^{1, m}, s^{2, m}, \ldots, s^{m, m}\right)$ of $\mathbb{R}_{+}^{m}$, where (1) $s^{m, m}=0$, (2) $s^{1, m} \geq s^{2, m} \geq \ldots \geq s^{m, m}$, and (3) $s^{1, m}>s^{m, m}$. Given a profile $P_{N} \in \mathcal{L}\left(A_{m}\right)^{n}$ together with a score vector $S^{m}$, the score of the alternative $x \in A_{m}$ in $P_{N}$ is $S^{m}\left(x, P_{N}\right)=\sum_{i \in N} s^{r_{i}\left(x, P_{N}\right), m}$, where $r_{i}\left(x, P_{N}\right)$ is the rank of $x$ in $P_{i}$. A SWF $\alpha$ is a scoring rule if there exists a sequence $\left\{S_{\alpha}^{m}\right\}_{m \geq 3}=\left\{S_{\alpha}^{1}, S_{\alpha}^{2}, S_{\alpha}^{3} \ldots\right\}$ of score vectors such that, for any $m, n \in \mathbb{N}$, for any $P_{N} \in \mathcal{L}\left(A_{m}\right)^{N}$, for any two alternatives $x, y \in A_{m}, x \alpha\left(P_{N}\right) y \Longleftrightarrow S_{\alpha}^{m}\left(x, P_{N}\right) \geq S_{\alpha}^{m}\left(y, P_{N}\right)$. We begin with the analysis of well-known scoring rules, namely the Borda rule, the plurality rule and the anti-plurality rule.

The Borda rule $\mathcal{B}$ is defined by: for any $m \in \mathbb{N}$, for any $k \in\{1, \ldots, m-1\}, s_{\mathcal{B}}^{k, m}=s_{\mathcal{B}}^{k+1, m}+1$. It is easily checked that $\mathcal{B}$ is not Kemeny-stable, hence not hyper-stable for $\mathcal{E}$. Indeed, consider the following profile $P_{N}$ involving 3 alternatives $a, b, c$ and 6 individuals, where the first row indicates the number of individuals sharing the same preference order

$$
P_{N}=\left(\begin{array}{lll}
3 & 1 & 2 \\
\hline a & c & c \\
b & b & a \\
c & a & b
\end{array}\right)
$$

Next, consider the following linearization $\dot{P}_{N}$ of $P_{N}^{\mathcal{K}}$ :

$$
\dot{P}_{N}=\left(\begin{array}{ccc}
3 & 1 & 2 \\
\hline a b c & c b a & c a b \\
b a c & c a b & c b a \\
a c b & b c a & a c b \\
b c a & b a c & a b c \\
c a b & a c b & b c a \\
c b a & a b c & b a c
\end{array}\right)
$$

Finally, $\mathcal{B}\left(P_{N}\right)=\{a c b\}=\Delta\left(\mathcal{B}\left(P_{N}\right)\right)$, whereas $S_{\mathcal{B}}^{6}\left(a c b, \dot{P}_{N}\right)=16<S_{\mathcal{B}}^{6}\left(a b c, \dot{P}_{N}\right)=19$ implies that $a c b \notin f_{\mathcal{B}}\left(\dot{P}_{N}\right)$. Since $\Delta\left(\mathcal{B}\left(P_{N}\right)\right) \cap f_{\mathcal{B}}\left(\dot{P}_{N}\right)=\varnothing$, then $\mathcal{B}$ is not Kemeny-stable.

The plurality rule is the scoring rule $\pi$, where, for any $m \in \mathbb{N}, s_{\pi}^{k, m}=0$ for any $k=2, \ldots, m$, and $s_{\pi}^{1, m}=1$. Consider an alteration $P_{N}^{\prime}$ of the profile $P_{N}$ above where the individual with preference $c b a$ changes to $b c a$. Then $\pi\left(P_{N}^{\prime}\right)=\{a c b\}$, while, for any linearization $\dot{P}_{N}^{\prime}$ of $P_{N}^{\prime K} f_{\pi}\left(\dot{P}_{N}^{\prime}\right)=\{a b c\}$. Hence, $\pi$ is not Kemeny-stable.

The anti-plurality rule is the scoring rule $\lambda$, where, for any $m \in \mathbb{N}, S_{\lambda}^{k, m}=1$ for any $1 \leq k \leq m-1$. Consider the following profile $P_{N} \in \mathcal{L}\left(A_{3}\right)^{15}$, where $\lambda\left(P_{N}\right)=\{a b c\}$, together with its associated Kemeny weak profile $P_{N}^{K}$ :

$$
P_{N}=\left(\begin{array}{ccccc}
3 & 2 & 3 & 3 & 4 \\
\hline a & a & b & c & c \\
b & c & a & a & b \\
c & b & c & b & a
\end{array}\right) \quad P_{N}^{K}=\left(\begin{array}{ccccc}
3 & 2 & 3 & 3 & 4 \\
\hline a b c & a c b & b a c & c a b & c b a \\
a c b, b a c & a b c, c a b & a b c, b c a & c b a, a c b & c a b, b c a \\
b c a, c a b & c b a, b a c & a c b, c b a & a b c, b c a & b a c, a c b \\
c b a & b c a & c a b & b a c & a b c
\end{array}\right)
$$


We conclude that, for all $P \in \mathcal{L}\left(A_{6}\right) /\{a b c\}, P \lambda\left(\dot{P}_{N}\right) a b c$ for all $\dot{P}_{N} \in \Delta\left(P_{N}^{K}\right)$. Thus, $a b c \notin f_{\lambda}\left(\dot{P}_{N}\right)$, which implies that $\lambda$ is not Kemeny-stable.

We state below four negative results about Kemeny-stable scoring rules. The key-ingredient of the proofs is the following Theorem, which characterizes Kemeny-stable scoring rules for 3 alternatives.

Theorem 1 A scoring rule $\alpha$ is Kemeny-stable only if $s_{\alpha}^{1,3}=2 \cdot s_{\alpha}^{2,3}>0$, and $s_{\alpha}^{1,6}=\frac{4}{3} s_{\alpha}^{2,6}=\frac{4}{3} s_{\alpha}^{3,6}=4 s_{\alpha}^{4,6}=$ $4 s_{\alpha}^{5,6}>s_{\alpha}^{6,6}=0$. Furthermore, this is also sufficient for a scoring rule to be Kemeny-stable for three alternative case.

A scoring rule $\alpha$ is non-truncated if there exists no $m \in \mathbb{N}$ and no $k \in\{2, \ldots, m-1\}$ such that $s_{\alpha}^{k, m}=0$ : the score vector defined for some number $m$ of alternatives gives a strictly positive score to any rank above the last one.

Theorem 2 There is no Kemeny-stable and non-truncated scoring rule.

A scoring rule $\alpha$ is strict-at-top if, for any $m \in \mathbb{N}, s_{\alpha}^{1, m}>s_{\alpha}^{2, m}$ : all score vectors give a score to the top-ranked alternative strictly higher than any other score. Typical examples of strict-at-top scoring rules are the plurality and the Borda rules. Note that any convex scoring rule is also strict-at-top ${ }^{11}$.

Theorem 3 There is no Kemeny-stable and strict-at-top scoring rule.

Since a unanimous scoring rule must be strict-at-top and non-truncated, we can state the following corollary of Theorems 2 and 3.

Theorem 4 There is no Kemeny-stable and unanimous scoring rule.

When enlarging the Kemeny domain $\mathcal{K}$ to the domain $\mathcal{S}$ of separable preference extensions, we get an even stronger negative result:

Theorem 5 No scoring rule is hyper-stable for $\mathcal{S}$.

\section{Condorcet social welfare functions}

We turn now to the analysis of Condorcet SWFs. We begin with some additional notations and definitions. Given a profile $P_{N} \in \mathcal{L}\left(A_{m}\right)^{n}$, where $n$ is odd, the majority tournament for $P_{N}$ is the complete and asymmetric binary relation $\mu\left(P_{N}\right)$ defined over $A_{m} \times A_{m}$ by: $\forall(x, y) \in A_{m} \times A_{m}, x$ $\mu\left(P_{N}\right) y \Leftrightarrow\left|\left\{i \in N: x P_{i} y\right\}\right|>\left|\left\{i \in N: y P_{i} x\right\}\right|$. Given any $P_{N}$, the Condorcet winner of $P_{N}$ is the element $C W\left(P_{N}\right) \in A_{m}$ such that $C W\left(P_{N}\right) \mu\left(P_{N}\right) a$ for all $a \in A_{m} / C W\left(P_{N}\right)$. A SWF $\alpha$ is Condorcet if, for any profile, $\alpha$ ranks the Condorcet winner at top whenever it exists.

We prove below the existence of a Condorcet SWF hyper-stable for $\mathcal{S}$. Beforehand, we show that three well-known Condorcet SWFs violate Kemeny stability. The Copeland solution is the SWF $\varphi$ defined by: $\forall m \in \mathbb{N}, \forall n \in 2 \mathbb{N}+1, \forall P_{N} \in \mathcal{L}\left(A_{m}\right)^{n}, \forall x, y \in A_{m}, x \varphi\left(P_{N}\right)$ y $\Leftrightarrow c\left(x, P_{N}\right) \geq$ $c\left(y, P_{N}\right)$, where $c\left(x, P_{N}\right)=\left|\left\{z \in A_{m}: x \mu\left(P_{N}\right) z\right\}\right|$. Consider the following profile $P_{N}$, together with the linearization $\dot{P}_{N}$ of $P_{N}^{K}$ :

\footnotetext{
${ }^{11}$ A scoring rule $\alpha$ is convex if, for any $m \in \mathbb{N}$, the score vector $S_{\alpha}^{m}=\left(s_{\alpha}^{1, m}, \ldots, s_{\alpha}^{m, m}\right)$ is such that $\left(s_{\alpha}^{1, m}-s_{\alpha}^{2, m}\right) \geq$ $\left(s_{\alpha}^{2, m}-s_{\alpha}^{3, m}\right) \geq \ldots \geq\left(s_{\alpha}^{m-1, m}-s_{\alpha}^{m, m}\right)$.
} 


$$
P_{N}=\left(\begin{array}{ccccc}
1 & 1 & 1 & 1 & 1 \\
\hline a & a & b & b & c \\
b & c & c & a & a \\
c & b & a & c & b
\end{array}\right) \quad \dot{P}_{N}=\left(\begin{array}{ccccc}
1 & 1 & 1 & 1 & 1 \\
\hline a b c & a c b & b c a & b a c & c a b \\
a c b & c a b & b a c & b c a & a c b \\
b a c & a b c & c b a & a b c & c b a \\
c a b & c b a & c a b & a c b & b c a \\
b c a & b a c & a b c & c b a & a b c \\
c b a & b c a & a c b & c a b & b a c
\end{array}\right)
$$

Then, we have $\varphi\left(P_{N}\right)=a b c$, while $c\left(a b c, \dot{P}_{N}\right)=3<c\left(a c b, \dot{P}_{N}\right)=4$ implies that $\Delta\left(\varphi\left(P_{N}\right)\right) \cap$ $f_{\varphi}\left(\dot{P}_{N}\right)=\varnothing$. Thus, $\varphi$ is not Kemeny-stable.

The Slater solution is the social welfare correspondence ${ }^{12} \beta$ defined by: $\forall m \in \mathbb{N}, \forall n \in 2 \mathbb{N}+1$, $\forall P_{N} \in \mathcal{L}\left(A_{m}\right)^{n}, \forall P \in \mathcal{L}\left(A_{m}\right), \beta\left(P_{N}\right)=\operatorname{ArgMin}_{P \in \mathcal{L}\left(A_{m}\right)} d_{K}\left(P, \mu\left(P_{N}\right)\right)$. A SWF $\alpha$ is Slater-consistent if, at any profile $P_{N}$, it always selects one linear order in $\beta\left(P_{N}\right)$. Consider the following profile $P_{N} \in \mathcal{L}\left(A_{8}\right)^{5}:$

$$
P_{N}=\left(\begin{array}{ccccc}
1 & 1 & 1 & 1 & 1 \\
\hline b & a & d & c & d \\
c & b & a & a & b \\
d & c & b & d & c \\
a & d & c & b & a \\
a^{\prime} & b^{\prime} & d^{\prime} & d^{\prime} & c^{\prime} \\
b^{\prime} & c^{\prime} & a^{\prime} & b^{\prime} & a^{\prime} \\
c^{\prime} & d^{\prime} & b^{\prime} & c^{\prime} & d^{\prime} \\
d^{\prime} & a^{\prime} & c^{\prime} & a^{\prime} & b^{\prime}
\end{array}\right)
$$

Define $X=\{a, b, c, d\}$ and $Y=\left\{a^{\prime}, b^{\prime}, c^{\prime}, d^{\prime}\right\}$ and consider the restrictions $\left.P_{N}\right|_{X}$ and $\left.P_{N}\right|_{Y}$ of $P_{N}$ to $X$ and $Y$ respectively. We have that $\mu\left(\left.P_{N}\right|_{X}\right)$ and $\mu\left(\left.P_{N}\right|_{Y}\right)$ are isomorphic. Moreover, we observe that (1) $a \mu\left(P_{N}\right) b \mu\left(P_{N}\right) c \mu\left(P_{N}\right) d \mu\left(P_{N}\right) a,(2) c \mu\left(P_{N}\right) a$, (3) $d \mu\left(P_{N}\right) b$, and (4) all alternatives in $X$ defeat all alternatives in $Y$. This ensures that $\beta\left(\left.P_{N}\right|_{X}\right)=\{c d a b\}$ and $\beta\left(\left.P_{N}\right|_{Y}\right)=\left\{c^{\prime} d^{\prime} a^{\prime} b^{\prime}\right\}$. Thus, $\beta\left(P_{N}\right)=\left\{c d a b c^{\prime} d^{\prime} a^{\prime} b^{\prime}\right\}$. Now, consider $Q=d b c a d^{\prime} b^{\prime} c^{\prime} a^{\prime}$. The next table gives the Kemeny distances between each of the 5 linear orders in $P=\left(P_{1}, \ldots, P_{5}\right)$ and respectively, $\beta\left(P_{N}\right)$ and $Q$ :

\begin{tabular}{ccc}
$P_{i}$ & $\beta\left(P_{N}\right)$ & $Q$ \\
\hline$P_{1}$ & $3+4$ & $2+5$ \\
$P_{2}$ & $4+3$ & $5+2$ \\
$P_{3}$ & $3+3$ & $2+2$ \\
$P_{4}$ & $1+3$ & $4+0$ \\
$P_{5}$ & $3+1$ & $0+4$
\end{tabular}.

It follows that in the Kemeny weak profile $P_{N^{\prime}}^{K} Q$ is strictly preferred to $\beta\left(P_{N}\right)$ by individual 3, while all other individuals are indifferent. Hence, there exists a linearization $\dot{P}_{N}$ of $P_{N}^{K}$ where $Q$ is

\footnotetext{
${ }^{12} \mathrm{~A}$ social welfare correspondence is a mapping $\delta$ from $\underset{n, m \in \mathbb{N}}{\cup} \mathcal{L}\left(A_{m}\right)^{n}$ to $\underset{m \in \mathbb{N}}{\cup} 2^{\mathcal{R}\left(A_{m}\right)}$ such that, for any $n, m \in \mathbb{N}$, for any $P_{N} \in \mathcal{L}\left(A_{m}\right)^{n}, \delta\left(P_{N}\right) \in 2^{\mathcal{R}\left(A_{m}\right)}$, where $2^{\mathcal{R}\left(A_{m}\right)}$ is the set of all non-empty subsets of weak orders over $A_{m}$.
} 
unanimously preferred to $\beta\left(P_{N}\right)$. Since the Slater solution is contained in the Pareto set, and since $\beta\left(P_{N}\right)$ is a singleton, we conclude that no Slater-consistent SWF is Kemeny-stable.

The Kemeny rule is the Condorcet social welfare correspondence $\omega$ defined by: $\forall P_{N}=\left(P_{1}, \ldots, P_{n}\right) \in$ $\mathcal{L}\left(A_{m}\right)^{n}, \forall P \in \mathcal{L}\left(A_{m}\right), \omega\left(P_{N}\right)=\operatorname{ArgMin}_{P \in \mathcal{L}\left(A_{m}\right)} \sum_{i \in N} d_{K}\left(P, P_{i}\right)$. A SWF $\alpha$ is Kemeny-consistent if, for any profile $P_{N}$, it always selects a linear order in $\omega\left(P_{N}\right)$. Consider the following profile $P_{N} \in \mathcal{L}\left(A_{3}\right)^{9}$ together with the linearization $\dot{P}_{N}$ of $P_{N}^{K}$ :

$$
P_{N}=\left(\begin{array}{ccc}
2 & 3 & 4 \\
\hline b & c & a \\
c & a & b \\
a & b & c
\end{array}\right) \dot{P}_{N}=\left(\begin{array}{ccc}
2 & 3 & 4 \\
\hline b c a & c a b & a b c \\
c b a & c b a & a c b \\
b a c & a c b & b a c \\
c a b & b c a & c a b \\
a b c & a b c & b c a \\
a c b & b a c & c b a
\end{array}\right)
$$

The reader will check that $\omega\left(P_{N}\right)=\{a b c\}$, whereas $\omega\left(\dot{P}_{N}\right)=\{(c a b)(a b c)(a c b)(b c a)(c b a)(b a c)\}$ which leads to $f_{\omega}\left(\dot{P}_{N}\right)=\{c a b\}$. Hence, there is no Kemeny-stable and Kemeny-consistent SWF.

We now establish the existence of a Condorcet and unanimous SWF which is hyper-stable for $\mathcal{S}$. The transitive closure $\theta\left(P_{N}\right)$ of $\mu\left(P_{N}\right)$ is defined by: $\forall x, y \in A_{m}, x \theta\left(P_{N}\right) y$ if and only if there exist $x_{1}, x_{2}, \ldots, x_{H} \in A_{m}$ such that $x \mu\left(P_{N}\right) x_{1}, x_{1} \mu\left(P_{N}\right) x_{2}, \ldots, x_{H} \mu\left(P_{N}\right) y$. Consider the SWF $\theta$, which maps every profile $P_{N} \in \cup_{m, n} \mathcal{L}\left(A_{m}\right)^{n}$ (where $n$ is odd) to the transitive closure $\theta\left(P_{N}\right)$ of $\mu\left(P_{N}\right)$. It is easily checked that $\theta$ is unanimous.

Theorem 6 is hyper-stable for $\mathcal{S}$.

\section{Discussion}

Our main result is that no unanimous scoring rule is Kemeny-stable, hence hyper-stable for the larger domain $\mathcal{S}$ of separable preference extensions. However, the transitive closure of the majority relation is a unanimous Condorcet SWF that is hyper-stable for $\mathcal{S}$.

Hyper-stability does not draw a clear border between scoring rules and Condorcet SWFs. Indeed, several Condorcet SWFs based on well-known tournament solutions, as well as the Kemeny SWF, are not Kemeny-stable. Characterizing the class of Condorcet SWFs hyper-stable for $\mathcal{S}$ is an open question worth being addressed. Another open problem is studying hyper-stability for non-unanimous scoring rules.

Further open questions relate to alternative concepts of hyper-stability.

\subsection{Alternative hyper-stability concepts}

Any strongly Condorcet ${ }^{13}$ SWF $\alpha$ violates the following property of hyper Condorcet-stability: A SWF $\alpha$ is hyper Condorcet-stable if $\forall n, m \in \mathbb{N}, \forall P_{N} \in \mathcal{L}\left(A_{m}\right)^{n}, \forall E \in \mathcal{S}^{n}, \alpha\left(P_{N}\right) \in \mathcal{L}\left(A_{m}\right) \Rightarrow$

\footnotetext{
${ }^{13}$ A strongly Condorcet $\alpha$ is such that for any $m \in \mathbb{N}$, for any $n \in 2 \mathbb{N}+1$ and for any $P_{N} \in \mathcal{L}\left(A_{m}\right)^{n}$ we have $\mu\left(P_{N}\right) \in \mathcal{L}\left(A_{m}\right)$ only if $\alpha\left(P_{N}\right)=\mu\left(P_{N}\right)$.
} 
$\left[\alpha\left(P_{N}\right)=C W\left(P_{N}^{E}\right)\right]$. To see why, consider the following profile $P_{N} \in \mathcal{L}\left(A_{m}\right)^{5}$, together with the Kemeny hyper-profile $\dot{P}_{N} \in \Delta\left(P_{N}^{K}\right)$ :

$$
P_{N}=\left(\begin{array}{lll}
1 & 1 & 1 \\
\hline a & a & b \\
b & c & c \\
c & b & a
\end{array}\right) \dot{P}_{N}=\left(\begin{array}{lll}
1 & 1 & 1 \\
\hline a b c & a c b & b c a \\
b a c & c a b & c b a \\
a c b & a b c & b a c \\
b c a & c b a & c a b \\
c a b & b a c & a b c \\
c b a & b c a & a c b
\end{array}\right)
$$

Since $\mu\left(P_{N}\right)=a b c$, then $\alpha\left(P_{N}\right)=a b c$ for any strongly Condorcet $\alpha$. However, $\alpha\left(P_{N}\right)$ is defeated in $\mu\left(\dot{P}_{N}\right)$ by cab. An interesting question is whether any strongly Condorcet $\alpha$ satisfies the following weaker version of hyper Condorcet-stability: $\forall n, m \in \mathbb{N}, \forall P_{N} \in \mathcal{L}\left(A_{m}\right)^{n}$ such that $\alpha\left(P_{N}\right) \in \mathcal{L}\left(A_{m}\right)$, there exists $E \in \mathcal{S}^{n}$ for which $\alpha\left(P_{N}\right)=C W\left(P_{N}^{E}\right)$.

Note that there are strongly Condorcet SWFs that are hyper-stable for $\mathcal{S}$. Define the SWF $\psi$ by: $\forall m, n \in \mathbb{N}, \forall P_{N} \in \mathcal{L}\left(A_{m}\right)^{n}, \psi\left(P_{N}\right)=\mu\left(P_{N}\right)$ if $\mu\left(P_{N}\right) \in \mathcal{L}\left(A_{m}\right)$, and otherwise, $a \psi\left(P_{N}\right) b$ and $b \psi\left(P_{N}\right) a$ for all $a, b \in A_{m}$. Then $\psi$ is hyper stable for $\mathcal{S}$. This is an immediate corollary of the following proposition:

Proposition 2 Let $P_{N} \in \mathcal{L}\left(A_{m}\right)^{n}$ be such that $\mu\left(P_{N}\right) \in \mathcal{L}\left(A_{m}\right)$. For any $E \in \mathcal{S}^{n}$, either $C W\left(P_{N}^{E}\right)$ does not exist, or $C W\left(P_{N}^{E}\right)=\mu\left(P_{N}\right)$.

Remark that, in the Kemeny hyper-profile $\dot{P}_{N}$ above, all three individual preferences are extended through the same linearization of the Kemeny weak order. This common linearization can be defined as a linear order over the permutations of the set $\{1,2,3\}$ of ranks. Indeed, given two orders $P$ and $Q=\left(a_{1} a_{2} \ldots a_{m}\right)$ in $\mathcal{L}\left(A_{m}\right)$, define $r_{P}(Q)=\left(r_{P}\left(a_{1}\right), \ldots, r_{P}\left(a_{m}\right)\right)$ by $\forall h=1, \ldots, m, r_{P}\left(a_{h}\right)=$ $\left|\left\{b \in A_{m}: b P a_{h}\right\}\right|+1$, that is, the rank given to $a_{h}$ in $P$. Moreover, given $P_{N}=\left(P_{1}, \ldots, P_{n}\right) \in \mathcal{L}\left(A_{m}\right)^{n}$ , we say that the hyper-profile $P_{N}^{E}=\left(e_{1}\left(P_{1}\right), \ldots, e_{n}\left(P_{n}\right)\right)$ is uniform if there exists a linear order $\succ$ over the permutations of $\{1, \ldots, m\}$ such that, for any $i=1, \ldots, n$, for any $Q, Q^{\prime} \in \mathcal{L}\left(A_{m}\right),\left[Q e^{i}\left(P_{i}\right)\right.$ $\left.Q^{\prime} \Leftrightarrow r_{P_{i}}(Q) \succ r_{P_{i}}\left(Q^{\prime}\right)\right]$. In the example above, $\succ$ is defined by: (123) $\succ(213) \succ(132) \succ(231)$ $\succ(312) \succ$ (321). We say that a SWF $\alpha$ is uniformly hyper-stable for $\mathcal{S}$ if $\forall n, m \in \mathbb{N}, \forall P_{N} \in \mathcal{L}\left(A_{m}\right)^{n}$, $\Delta\left(\alpha\left(P_{N}\right)\right) \cap f_{\alpha}\left(P_{N}^{E}\right) \neq \varnothing$ for all uniform hyper-profiles $P_{N}^{E}$ with $E \in \mathcal{S}^{n}$.

As a first step towards a complete study of uniform hyper-stability, we remark that the Borda rule $\mathcal{B}$ is not uniformly hyper-stable. To see why, consider the following profile $P_{N}$, together with the Kemeny hyper-profile $\dot{P}_{N} \in \Delta\left(P_{N}^{K}\right)$ :

$$
P_{N}=\left(\begin{array}{ccc}
1 & 1 & 2 \\
\hline a & b & c \\
b & a & b \\
c & c & a
\end{array}\right) \dot{P}_{N}=\left(\begin{array}{lll}
1 & 1 & 2 \\
\hline a b c & b a c & c b a \\
a c b & b c a & c a b \\
b a c & a b c & b c a \\
c a b & c b a & a c b \\
b c a & a c b & b a c \\
c b a & c a b & a b c
\end{array}\right)
$$


We get $\mathcal{B}\left(P_{N}\right)=b c a$. Moreover, $\dot{P}_{N}$ is uniform (to see why, consider (123) $\succ(132) \succ(213) \succ$ $(312) \succ(231) \succ(321))$. Finally, $S_{\mathcal{B}}^{6}\left(b c a, \dot{P}_{N}\right)=11<S_{\mathcal{B}}^{6}\left(c b a, \dot{P}_{N}\right)=12$.

\section{References}

Binmore K (1975) An example in group preference. Journal of Economic Theory 10:377-385

Bossert W, Sprumont Y (2012) Strategy-proof preference aggregation. Cahier $d u$ CIREQ No 122012, Université de Montréal

Bossert W, Storcken T (1992) Strategy-proofness of social welfare functions: the use of the Kemeny distance between preference orderings. Social Choice and Welfare 9:345-360

Duddy C, Perote-Pena J, Piggins A (2010) Manipulating an aggregation rule under ordinally fuzzy preferences. Social Choice and Welfare 34:411-428

Igersheim H (2007) Du paradoxe libéral-parétien à un concept de métaclassement des préférences. Recherches économiques de Louvain 73:173-192

Jeffrey RC (1974) Preferences among preferences. The Journal of Philosophy 13:377-391

Koray S (2000) Self-selective social choice functions verify Arrow and Gibbard-Satterthwaite theorems. Econometrica 68:981-995

Koray S, Unel B (2003) Characterization of self-selective social choice functions on the tops-only domain. Social Choice and Welfare 20:495-507

Koray S, Slinko A (2008) Self-selective social choice functions. Social Choice and Welfare 31:129-149

Laffond G, Lainé J (2000) Majority voting on orders. Theory and Decision, 49:251-289

McPherson MS (1982) Mill's moral theory and the problem of preference change. Ethics 92:252-273

Sen AK (1970) The impossibility of a Paretian liberal. Journal of Political Economy 78:152-157

Sen AK (1974) Choice, orderings and morality. in S. Korner (ed.) Practical Reason, Blackwell, Oxford

Sen AK (1977) Rational fools: A critique of behavioral foundations of economic theory. Philosophy and Public Affairs 6:317-344

\section{Appendices}

\section{A Proofs}

\section{A.1 Proof of Proposition 1}

Let $\alpha$ be a neutral SFW that is SW self-selective for some domain $\mathcal{E}$. Pick up any profile $P_{N} \in \mathcal{L}\left(A_{m}\right)^{n}$ where $\Delta\left(\alpha\left(P_{N}\right)\right)=\left\{Q_{1}, \ldots, Q_{H}\right\}$, together with any $E=\left(e_{1}, \ldots, e_{n}\right) \in \mathcal{E}^{n}$. Consider the set of SWFs $\mathcal{A}=\left\{\alpha_{1}, \alpha_{2}, \ldots, \alpha_{H}, \rho_{1}, \ldots, \rho_{m !-H}\right\}$ such that:

$-\alpha_{1}\left(P_{N}\right)=Q_{1}, \ldots, \alpha_{H}\left(P_{N}\right)=Q_{H}$

$-\forall k \neq k^{\prime} \in\{1, \ldots, m !-H\}, \alpha_{k}\left(P_{N}\right) \neq \alpha_{k^{\prime}}\left(P_{N}\right)$

$-\cup_{1 \leq k \leq m !-H} \rho_{k}\left(P_{N}\right)=\mathcal{L}\left(A_{m}\right)-\Delta\left(\alpha\left(P_{N}\right)\right)$ 
Since all elements of $\mathcal{A}$ are strict $\mathrm{SWFs}$, then $\mathcal{A}$ is a strict selection. Moreover, all elements of $\mathcal{A}$ having different outcomes from $P_{N}$, then $P_{N}^{E \mathcal{A}}$ is a profile of linear orders over $\mathcal{A}$. Furthermore, $\left[\cup_{1 \leq h \leq H} \alpha_{h}\left(P_{N}\right)\right] \cup\left[\cup_{1 \leq k \leq m !-1} \rho_{k}\left(P_{N}\right)\right]=\mathcal{L}\left(A_{m}\right)$ implies that $P_{N}^{E \mathcal{A}}$ is a profile over all $m$ ! linear orders, so that $L(\alpha) \cap \mathcal{A}=L(\alpha)$. It follows from definition that $P_{N}^{E \mathcal{A}}$ is isomorphic to $P_{N}^{E}$. Since $\alpha$ is $\mathrm{SW}$ self-selective for $E$, then $L(\alpha) \cap f_{\alpha}\left(\widetilde{P}_{N}^{E \mathcal{A}}\right) \neq \varnothing$. Since $P_{N}^{E \mathcal{A}^{*}}$ is isomorphic to $P_{N}^{E}$, the neutrality of $\alpha$ ensures that $\Delta\left(\alpha\left(P_{N}\right)\right) \cap f_{\alpha}\left(P_{N}^{E}\right) \neq \varnothing$ and the conclusion follows.

\section{A.2 Proof of Theorem 1}

We first prove the following three propositions, each providing a necessary condition for Kemenystability.

Proposition 3 A scoring rule $\alpha$ is Kemeny-stable only if $s_{\alpha}^{2,3}>0$ and $s_{\alpha}^{1,6}>s_{\alpha}^{2,6}=s_{\alpha}^{3,6}>s_{\alpha}^{4,6}=s_{\alpha}^{5,6}>$ $s_{\alpha}^{6,6}=0$.

Proposition 4 A scoring rule $\alpha$ is Kemeny-stable only if $s_{\alpha}^{1,6}=s_{\alpha}^{2,6}+s_{\alpha}^{4,6}$.

Proposition 5 A scoring rule $\alpha$ is Kemeny-stable only if $s_{\alpha}^{1,3}=2 s_{\alpha}^{2,3}$.

\section{A.2.1 Proof of Proposition 3}

The proof is organized in six 6 intermediate lemmas:

Lemma 1 If $\alpha$ is a Kemeny-stable scoring rule, then $s_{\alpha}^{2,3}>0$.

Proof: Suppose that $s_{\alpha}^{2,3}=0$, and consider the following profile $P_{N} \in \mathcal{L}\left(A_{3}\right)^{n_{1}+n_{2}+n_{3}+n_{4}}$, where $n_{1}>n_{2}>n_{3}+n_{4}$, together with the following linearization $\dot{P}_{N}$ of $P_{N}^{K}$ :

$$
P_{N}=\left(\begin{array}{cccc}
n_{1} & n_{2} & n_{3} & n_{4} \\
\hline a & b & c & c \\
c & c & a & b \\
b & a & b & a
\end{array}\right) \dot{P}_{N}=\left(\begin{array}{cccc}
n_{1} & n_{2} & n_{3} & n_{4} \\
\hline a c b & b c a & c a b & c b a \\
c a b & b a c & a c b & c a b \\
a b c & c b a & c b a & b c a \\
b a c & c a b & b c a & a c b \\
c b a & a b c & a b c & b a c \\
b c a & a c b & b a c & a b c
\end{array}\right)
$$

It follows that $\Delta\left(\alpha\left(P_{N}\right)\right)=\{a b c\}$. Kemeny-stability requires that $S_{\alpha}^{6}\left(a b c, \dot{P}_{N}\right)=n_{1} s_{\alpha}^{3,6}+\left(n_{2}+\right.$ $\left.n_{3}\right) s_{\alpha}^{5,6} \geq S_{\alpha}^{6}\left(c a b, \dot{P}_{N}\right)=\left(n_{1}+n_{4}\right) s_{\alpha}^{2,6}+n_{2} s_{\alpha}^{4,6}+n_{3} s_{\alpha}^{1,6}$, hence that $n_{1}\left(s_{\alpha}^{3,6}-s_{\alpha}^{2,6}\right)+n_{2}\left(s_{\alpha}^{5,6}-s_{\alpha}^{4,6}\right)+$ $n_{3}\left(s_{\alpha}^{5,6}-s_{\alpha}^{1,6}\right) \geq n_{4} s_{\alpha}^{2,6}$, which is clearly impossible

Lemma 2 If $\alpha$ is a Kemeny-stable scoring rule, then $s_{\alpha}^{2,6}=s_{\alpha}^{3,6}$ and $s_{\alpha}^{4,6}=s_{\alpha}^{5,6}$.

Proof: Suppose first that $s_{\alpha}^{1,3}>2 s_{\alpha}^{2,3}$, and consider $P_{N} \in \mathcal{L}\left(A_{3}\right)^{4}$, and $\dot{P}_{N} \in \Delta\left(P_{N}^{K}\right)$ : 


$$
P_{N}=\left(\begin{array}{cccc}
1 & 1 & 1 & 1 \\
\hline a & a & b & c \\
b & c & c & b \\
c & b & a & a
\end{array}\right) \dot{P}_{N}=\left(\begin{array}{cccc}
1 & 1 & 1 & 1 \\
\hline a b c & a c b & b c a & c b a \\
b a c & c a b & c b a & b c a \\
a c b & a b c & b a c & c a b \\
b c a & c b a & c a b & b a c \\
c a b & b a c & a b c & a c b \\
c b a & b c a & a c b & a b c
\end{array}\right)
$$

Since $S_{\alpha}^{3}\left(a, P_{N}\right)=2 s_{\alpha}^{1,3}$, and $S_{\alpha}^{3}\left(b, P_{N}\right)=S_{\alpha}^{3}\left(c, P_{N}\right)=s_{\alpha}^{1,3}+2 s_{\alpha}^{2,3}$, then $\Delta\left(\alpha\left(P_{N}\right)\right)=\{a b c, a c b\}$. Moreover, we have (1) $S_{\alpha}^{6}\left(a b c, \dot{P}_{N}\right)=S_{\alpha}^{3}\left(a c b, \dot{P}_{N}\right)=s_{\alpha}^{1,6}+s_{\alpha}^{3,6}+s_{\alpha}^{5,6}$, and (2) $S_{\alpha}^{6}\left(b c a, \dot{P}_{N}\right)=s_{\alpha}^{1,6}+$ $s_{\alpha}^{2,6}+s_{\alpha}^{4,6}$. Kemeny stability implies from (1) and (2) that $s_{\alpha}^{3,6}+s_{\alpha}^{5,6} \geq s_{\alpha}^{2,6}+s_{\alpha}^{4,6}(3)$, which in turn leads to $s_{\alpha}^{2,6}=s_{\alpha}^{3,6}$ and $s_{\alpha}^{4,6}=s_{\alpha}^{5,6}$.

Suppose now that $s_{\alpha}^{1,3}<2 s_{\alpha}^{2,3}$, and consider the same profile $P_{N}$ above and the hyper-profile $\dot{P}_{N}^{\prime} \in \Delta\left(P_{N}^{K}\right)$ obtained from $\dot{P}_{N}$ by switching in each order alternatives respectively ranked (1) second and third, and (2) fourth and fifth. We get $\Delta\left(\alpha\left(P_{N}\right)\right)=\{b c a, c b a\}$, and we reach the same conclusion as above by a symmetric argument. Finally, suppose that $s_{\alpha}^{1,3}=2 s_{\alpha}^{2,3}$, and consider the profile $P_{N} \in$ $\mathcal{L}\left(A_{3}\right)^{4 Z-1}$ below, where $Z>1$, together with the Kemeny weak profile $P_{N}^{K}$ :

$$
P_{N}=\left(\begin{array}{cccc}
Z & Z & Z-1 & Z \\
\hline a & b & c & c \\
b & a & b & a \\
c & c & a & b
\end{array}\right) P_{N}^{K}=\left(\begin{array}{cccc}
Z & Z & Z-1 & Z \\
\hline a b c & b a c & c b a & c a b \\
a c b, b a c & a b c, b c a & b c a, c a b & c b a, a c b \\
b c a, c a b & c b a, a c b & a c b, b a c & a b c, b c a \\
c b a & c a b & a b c & b a c
\end{array}\right)
$$

Then $\alpha\left(P_{N}\right)=a b c$. Moreover, there exists $\dot{P}_{N} \in \Delta\left(P_{N}^{K}\right)$ such that $S_{\alpha}^{6}\left(a b c, \dot{P}_{N}\right)=Z\left(s_{\alpha}^{1,6}+s_{\alpha}^{3,6}+s_{\alpha}^{5,6}\right)$ and $S_{\alpha}^{6}\left(c a b, \dot{P}_{N}\right)=Z s_{\alpha}^{1,6}+(Z-1) s_{\alpha}^{2,6}+Z s_{\alpha}^{4,6}$. Kemeny stability requires that $s_{\alpha}^{3,6}+s_{\alpha}^{5,6} \geq \frac{Z-1}{Z} s_{\alpha}^{2,6}+s_{\alpha}^{4,6}$ for all $Z>1$. Thus, $s_{\alpha}^{2,6}+s_{\alpha}^{4,6} \leq s_{\alpha}^{3,6}+s_{\alpha}^{5,6}$, and hence $s_{\alpha}^{2,6}=s_{\alpha}^{3,6}$ and $s_{\alpha}^{4,6}=s_{\alpha}^{5,6}$

We assume in the sequel that $\alpha$ is such that $s_{\alpha}^{2,6}=s_{\alpha}^{3,6}$ and $s_{\alpha}^{4,6}=s_{\alpha}^{5,6}$ (property $(*)$ ). Clearly, $(*)$ implies that given any profile $P_{N}$ over 3 alternatives, given any Kemeny-stable SWF $\alpha$, one has $\alpha\left(\dot{P}_{N}\right)=\alpha\left(\widetilde{P_{N}}\right)$ for any two $\forall \dot{P}_{N}, \widetilde{P_{N}} \in \Delta\left(P_{N}^{K}\right)$.

Lemma 3 If $\alpha$ is a Kemeny-stable scoring rule, then $\left[s_{\alpha}^{1,6}=s_{\alpha}^{2,6}\right] \Rightarrow\left[s_{\alpha}^{4,6}=s_{\alpha}^{5,6}>0\right]$.

Proof: Consider the following $P_{N} \in \mathcal{L}\left(A_{3}\right)^{3 Z+W}$ below, where $Z, W \geq 1$ are chosen such that $W<\frac{s_{\alpha}^{2,3}}{s_{\alpha}^{1,3}} Z:$

$$
P_{N}=\left(\begin{array}{cccc}
Z & Z & Z & W \\
\hline a & b & c & a \\
b & a & b & c \\
c & c & a & b
\end{array}\right)
$$

Then $\alpha\left(P_{N}\right)=$ bac. Furthermore, using $(*)$ together with Kemeny stability and $s_{\alpha}^{1,6}=s_{\alpha}^{2,6}$, one must have $S_{\alpha}^{6}\left(b a c, \dot{P}_{N}\right)=2 Z s_{\alpha}^{1,6}+(Z+W) s_{\alpha}^{5,6} \geq S_{\alpha}^{6}\left(a b c, \dot{P}_{N}\right)=(2 Z+W) s_{\alpha}^{1,6}$. Thus, $s_{\alpha}^{1,6} \leq \frac{Z+W}{W} s_{\alpha}^{5,6}$. Finally, since $s_{\alpha}^{1,6}>0$, then $s_{\alpha}^{5,6}>0 \square$ 
Lemma 4 If $\alpha$ is a Kemeny-stable scoring rule, then $\left[s_{\alpha}^{1,6}=s_{\alpha}^{2,6}\right] \Rightarrow\left[2 s_{\alpha}^{1,3}=3 s_{\alpha}^{2,3}\right]$.

Proof: Define the two profiles $P_{N} \in \mathcal{L}\left(A_{3}\right)^{5}$ and $P_{N}^{\prime} \in \mathcal{L}\left(A_{3}\right)^{3 Z+1}$, where $Z>1$, as follows:

$$
P_{N}=\left(\begin{array}{cccc}
2 & 1 & 1 & 1 \\
\hline a & a & c & b \\
b & c & b & c \\
c & b & a & a
\end{array}\right) P_{N}^{\prime}=\left(\begin{array}{ccc}
2 Z & 1 & Z \\
\hline a & c & c \\
b & a & b \\
c & b & a
\end{array}\right)
$$

Suppose first that $2 s_{\alpha}^{1,3}>3 s_{\alpha}^{2,3}$. It follows from $2 s_{\alpha}^{1,3}>3 s_{\alpha}^{2,3}$ that $\alpha\left(P_{N}\right)=a b c$. Using $(*)$, we have $s_{\alpha}^{1,6}=s_{\alpha}^{2,6}=s_{\alpha}^{3,6} \geq s_{\alpha}^{4,6}=s_{\alpha}^{5,6}$. Hence, $\forall \dot{P}_{N} \in \Delta\left(P_{N}^{K}\right), s_{\alpha}^{6}\left(a b c, \dot{P}_{N}\right)=3 s_{\alpha}^{1,6}+s_{\alpha}^{5,6}$, and $S_{\alpha}^{6}\left(b a c, \dot{P}_{N}\right)=$ $3 s_{\alpha}^{1,6}+2 s_{\alpha}^{5,6}$. Since Kemeny-stability requires $S_{\alpha}^{6}\left(a b c, \dot{P}_{N}\right) \geq S_{\alpha}^{6}\left(b a c, \dot{P}_{N}\right)$, then we get $s_{\alpha}^{5,6}=0$, in contradiction with Lemma 3.

Similarly, suppose that $2 s_{\alpha}^{1,3}<3 s_{\alpha}^{2,3}$. From $0<2 s_{\alpha}^{1,3}<3 s_{\alpha}^{2,3}$, we get that $\alpha\left(P_{N}^{\prime}\right)=$ bac for $Z$ large enough. Moreover, $\forall \dot{P}_{N} \in \Delta\left(P_{N}^{\prime K}\right), S_{\alpha}^{6}\left(b a c, \dot{P}_{N}\right)=Z\left(2 s_{\alpha}^{1,6}+s_{\alpha}^{5,6}\right)<S_{\alpha}^{6}\left(a c b, \dot{P}_{N}\right)=Z\left(2 s_{\alpha}^{1,6}+s_{\alpha}^{5,6}\right)+s_{\alpha}^{1,6}$, in contradiction with Kemeny stability

Lemma 5 If $\alpha$ is a Kemeny-stable scoring rule, then $s_{\alpha}^{1,6}>s_{\alpha}^{2,6}$.

Proof: Suppose that $s_{\alpha}^{1,6}=s_{\alpha}^{2,6}$. From Lemma 3 and 4 together with $(*)$, we have $s_{\alpha}^{1,6}=s_{\alpha}^{2,6}=s_{\alpha}^{3,6}$, $2 s_{\alpha}^{1,3}=3 s_{\alpha}^{2,3}$, and $s_{\alpha}^{4,6}=s_{\alpha}^{5,6}>0$. Then, consider the following profile $P_{N} \in \mathcal{L}\left(A_{3}\right)^{4}$ :

$$
P_{N}=\left(\begin{array}{cccc}
2 & 1 & 1 & 1 \\
\hline a & b & c & c \\
b & a & b & a \\
c & c & a & b
\end{array}\right)
$$

Since $S_{\alpha}^{3}\left(a, P_{N}\right)=2 s_{\alpha}^{1,3}+2 s_{\alpha}^{2,3}, S_{\alpha}^{3}\left(b, P_{N}\right)=s_{\alpha}^{1,3}+3 s_{\alpha}^{2,3}$, and $S_{\alpha}^{3}\left(c, P_{N}\right)=2 s_{\alpha}^{1,3}$, then, using Lemma 1 and Lemma 4, $\alpha\left(P_{N}\right)=a b c$. From Kemeny-stability, we have that for any $\dot{P}_{N} \in \Delta\left(P_{N}^{K}\right), S_{\alpha}^{6}\left(a b c, \dot{P}_{N}\right)=$ $3 s_{\alpha}^{1,6}+s_{\alpha}^{5,6} \geq S_{\alpha}^{6}\left(a c b, \dot{P}_{N}\right)=3 s_{\alpha}^{1,6}+2 s_{\alpha}^{5,6}$. But this implies that $s_{\alpha}^{5,6}=0$, in contradiction with Lemma $3 \square$

Lemma 6 If $\alpha$ is a Kemeny-stable scoring rule, then $s_{\alpha}^{3,6}>s_{\alpha}^{4,6}$.

Proof: Suppose that $s_{\alpha}^{3,6}=s_{\alpha}^{4,6}$. It follows from Lemma 2 together with Lemma 5 that $s_{\alpha}^{1,6}>s_{\alpha}^{2,6}=$ $s_{\alpha}^{3,6}=s_{\alpha}^{4,6}=s_{\alpha}^{5,6} \geq s_{\alpha}^{6,6}=0$. Using Lemma 1, we get the following possible cases:

Case 1: $s_{\alpha}^{1,3}=s_{\alpha}^{2,3}>0$

Consider the 4 following profiles:

$$
P_{N}=\left(\begin{array}{lllll}
3 & 2 & 3 & 3 & 4 \\
\hline a & a & b & c & c \\
b & c & a & a & b \\
c & b & c & b & a
\end{array}\right) P_{N}^{\prime}=\left(\begin{array}{ccc}
1 & 3 & 1 \\
\hline a & b & c \\
b & a & a \\
c & c & b
\end{array}\right) P_{N}^{\prime \prime \prime}=\left(\begin{array}{cccc}
1 & 1 & 1 & 1 \\
\hline a & a & b & c \\
b & c & a & b \\
c & b & c & a
\end{array}\right) P_{N}^{\prime \prime \prime}=\left(\begin{array}{ccc}
2 & 2 & 1 \\
\hline a & b & c \\
c & a & a \\
b & c & b
\end{array}\right)
$$


If $s_{\alpha}^{2,6}>0$, then $\alpha\left(P_{N}\right)=a b c$. Since $S_{\alpha}^{6}\left(a b c, \dot{P}_{N}\right)$

$=3 s_{\alpha}^{1,6}+8 s_{\alpha}^{2,6}<S_{\alpha}^{6}\left(c b a, \dot{P}_{N}\right)=4 s_{\alpha}^{1,6}+8 s_{\alpha}^{2,6}$ for all $\dot{P}_{N} \in \Delta\left(P_{N}^{K}\right)$, then $\alpha$ is not Kemeny-stable. If $s_{\alpha}^{2,6}=0$, then $\alpha\left(P_{N}^{\prime}\right)=a b c$. Since $f^{\alpha}\left(\dot{P}_{N}\right)=\{b a c\}$ for all $\dot{P}_{N} \in \Delta\left(P_{N}^{\prime K}\right)$, then $\alpha$ is not Kemeny-stable.

Case $2: s_{\alpha}^{1,3}>s_{\alpha}^{2,3}>0$

If $s_{\alpha}^{2,6}>0$, then $\alpha\left(P_{N}^{\prime \prime}\right)=a b c$. Since $S_{\alpha}^{6}\left(a b c, \dot{P}_{N}\right)$ $=s_{\alpha}^{1,6}+2 s_{\alpha}^{2,6}<S_{\alpha}^{6}\left(b a c, \dot{P}_{N}\right)=s_{\alpha}^{1,6}+3 s_{\alpha}^{2,6}$ for all $\dot{P}_{N} \in \Delta\left(P_{N}^{\prime \prime K}\right)$, then $\alpha$ is not Kemeny-stable. Finally, if $s_{\alpha}^{2,6}=0$, then $\alpha\left(P_{N}^{\prime \prime \prime}\right)=a b c$. Since $S_{\alpha}^{6}\left(a b c, \dot{P}_{N}\right)=0<S_{\alpha}^{6}\left(a c b, \dot{P}_{N}\right)=2 s_{\alpha}^{1,6}$ for all $\dot{P}_{N} \in \Delta\left(P_{N}^{\prime \prime \prime} K\right)$, then $\alpha$ is not Kemeny-stable.

Thus, Kemeny-stability requires that $s_{\alpha}^{3,6}>s_{\alpha}^{4,6}$

By combining the six lemmas above, we get that any Kemeny-stable scoring rule $\alpha$ must satisfy (1) $s_{\alpha}^{1,6}>s_{\alpha}^{2,6}=s_{\alpha}^{3,6}>s_{\alpha}^{4,6}=s_{\alpha}^{5,6} \geq 0=s_{\alpha}^{6,6}$, and (2) $s_{\alpha}^{1,3} \geq s_{\alpha}^{2,3}>0=s_{\alpha}^{3,3}$, hence Proposition 3.

\section{A.2.2 Proof of Proposition 4}

Suppose that $s_{\alpha}^{1,3}>2 s_{\alpha}^{2,3}$, and consider profiles $P_{N}, P_{N}^{\prime} \in \mathcal{L}\left(A_{3}\right)^{4}$ below:

$$
P_{N}=\left(\begin{array}{llll}
1 & 1 & 1 & 1 \\
\hline a & a & b & c \\
b & c & c & b \\
c & b & a & a
\end{array}\right) P_{N}^{\prime}=\left(\begin{array}{ccc}
1 & 2 & 1 \\
\hline a & a & b \\
b & c & c \\
c & b & a
\end{array}\right)
$$

Since $S_{\alpha}^{3}\left(a, P_{N}\right)=2 s_{\alpha}^{1,3}$ and $S_{\alpha}^{3}\left(b, P_{N}\right)=S_{\alpha}^{3}\left(c, P_{N}\right)=s_{\alpha}^{1,3}+2 s_{\alpha}^{2,3}$, then $s_{\alpha}^{1,3}>2 s_{\alpha}^{2,3} \Rightarrow \Delta\left(\alpha\left(P_{N}\right)=\right.$ $\{a b c, a c b\}$. Using Proposition 3, we get that for any $\dot{P}_{N} \in \Delta\left(P_{N}^{K}\right), S_{\alpha}^{6}\left(a b c, \dot{P}_{N}\right)=s_{\alpha}^{1,6}+s_{\alpha}^{2,6}+s_{\alpha}^{5,6}$, while $S_{\alpha}^{6}\left(b a c, \dot{P}_{N}\right)=2 s_{\alpha}^{2,6}+2 s_{\alpha}^{5,6}$. Therefore, Kemeny-stability requires $s_{\alpha}^{1,6} \geq s_{\alpha}^{2,6}+s_{\alpha}^{5,6}$. Similarly, since $S_{\alpha}^{3}\left(a, P_{N}^{\prime}\right)=3 s_{\alpha}^{1,3}, S_{\alpha}^{3}\left(b, P_{N}^{\prime}\right)=s_{\alpha}^{1,3}+s_{\alpha}^{2,3}$ and $S_{\alpha}^{3}\left(c, P_{N}^{\prime}\right)=3 s_{\alpha}^{2,3}$, then $s_{\alpha}^{1,3}>2 s_{\alpha}^{2,3} \Rightarrow \alpha\left(P_{N}^{\prime}\right)=a b c$. For any $\dot{P}_{N}^{\prime} \in \Delta\left(P_{N}^{\prime K}\right), S_{\alpha}^{6}\left(a b c, \dot{P}_{N}^{\prime}\right)=s_{\alpha}^{1,6}+2 s_{\alpha}^{2,6}+s_{\alpha}^{5,6}$, while $S_{\alpha}^{6}\left(a c b, \dot{P}_{N}^{\prime}\right)=2 s_{\alpha}^{1,6}+s_{\alpha}^{2,6}$. Thus, Kemenystability requires $s_{\alpha}^{1,6} \leq s_{\alpha}^{2,6}+s_{\alpha}^{5,6}$. Therefore, if $s_{\alpha}^{1,3}>2 s_{\alpha}^{2,3}$, then $s_{\alpha}^{1,6}=s_{\alpha}^{2,6}+s_{\alpha}^{5,6}$.

Suppose that $s_{\alpha}^{1,3}<2 s_{\alpha}^{2,3}$, and consider profiles $\widetilde{P}_{N} \in \mathcal{L}\left(A_{3}\right)^{5 Z+1}$, where $Z>1$, and $\bar{P}_{N} \in \mathcal{L}\left(A_{3}\right)^{4}$ below:

$$
\widetilde{P}_{N}=\left(\begin{array}{cccc}
2 Z & Z+1 & Z & Z \\
\hline a & c & c & b \\
b & b & a & c \\
c & a & b & a
\end{array}\right) \bar{P}_{N}=\left(\begin{array}{cccc}
1 & 1 & 1 & 1 \\
\hline a & a & b & c \\
b & c & c & b \\
c & b & a & a
\end{array}\right)
$$

Since $S_{\alpha}^{3}\left(a, \widetilde{P}_{N}\right)=2 Z s_{\alpha}^{1,3}+Z s_{\alpha}^{2,3}, S_{\alpha}^{3}\left(b, \widetilde{P}_{N}\right)=Z s_{\alpha}^{1,3}+(3 Z+1) s_{\alpha}^{2,3}$ and $S_{\alpha}^{3}\left(c, \widetilde{P}_{N}\right)=(2 Z+1) s_{\alpha}^{1,3}+$ $Z s_{\alpha}^{2,3}$, then if $Z$ is chosen large enough, $s_{\alpha}^{1,3}>2 s_{\alpha}^{2,3} \Rightarrow \alpha\left(P_{N}\right)=b c a$. Moreover, using again Proposition 3, Kemeny-stability implies that for any $Z>1$ and any $\dot{P}_{N} \in \Delta\left(\widetilde{P}_{N}^{K}\right), S_{\alpha}^{6}\left(b c a, \dot{P}_{N}\right) \geq S_{\alpha}^{6}\left(a b c, \dot{P}_{N}\right)$. Thus, $Z s_{\alpha}^{1,6}+(Z+1) s_{\alpha}^{2,6}+3 Z s_{\alpha}^{5,6} \geq 2 Z\left(s_{\alpha}^{1,6}+s_{\alpha}^{5,6}\right)$, and therefore $s_{\alpha}^{1,6} \leq\left(1+\frac{1}{Z}\right) s_{\alpha}^{2,6}+s_{\alpha}^{5,6}$ for all $Z>1$, leading to $s_{\alpha}^{1,6} \leq s_{\alpha}^{2,6}+s_{\alpha}^{5,6}$. Similarly, we get $\Delta\left(\alpha\left(\bar{P}_{N}\right)\right)=\{b c a, c b a\}$, while for any $\dot{P}_{N} \in \Delta\left(\bar{P}_{N}^{K}\right)$, $S_{\alpha}^{6}\left(b c a, \dot{P}_{N}\right)=S_{\alpha}^{6}\left(c b a, \dot{P}_{N}\right)=s_{\alpha}^{1,6}+s_{\alpha}^{2,6}+s_{\alpha}^{5,6}$, while $S_{\alpha}^{6}\left(b a c, \dot{P}_{N}\right)=2 s_{\alpha}^{2,6}+2 s_{\alpha}^{5,6}$. Thus, Kemenystability implies $s_{\alpha}^{1,6} \geq s_{\alpha}^{2,6}+s_{\alpha}^{5,6}$. Therefore, if $s_{\alpha}^{1,3}<2 s_{\alpha}^{2,3}$, then $s_{\alpha}^{1,6}=s_{\alpha}^{2,6}+s_{\alpha}^{5,6}$.

Finally, suppose that $s_{\alpha}^{1,3}=2 s_{\alpha}^{2,3}$ and consider $Q_{N} \in \mathcal{L}\left(A_{3}\right)^{4 Z+3}$ and $Q_{N}^{\prime} \in \mathcal{L}\left(A_{3}\right)^{10 Z+1}$, where $Z>1$ : 


$$
Q_{N}=\left(\begin{array}{cccc}
Z & Z+1 & Z+1 & Z+2 \\
\hline a & b & b & c \\
c & c & a & a \\
b & a & c & b
\end{array}\right) Q_{N}^{\prime}=\left(\begin{array}{ccccc}
3 Z & 3 Z+1 & 2 Z & 2 Z \\
\hline a & b & b & c \\
c & c & a & a \\
b & a & c & b
\end{array}\right)
$$

Since $s_{\alpha}^{1,3}=2 s_{\alpha}^{2,3}$, then $\alpha\left(Q_{N}\right)=c b a$. From Proposition 3, one has for any $\dot{P}_{N} \in \Delta\left(Q_{N}^{K}\right)$ that $S_{\alpha}^{6}\left(c b a, \dot{P}_{N}\right)=(2 Z+3) s_{\alpha}^{2,6}+(2 Z+1) s_{\alpha}^{5,6}$ and $S_{\alpha}^{6}\left(a c b, \dot{P}_{N}\right)=Z s_{\alpha}^{1,6}+(Z+2) s_{\alpha}^{2,6}+(Z+1) s_{\alpha}^{5,6}$. Kemenystability implies $s_{\alpha}^{1,6} \leq\left(1+\frac{1}{z}\right) s_{\alpha}^{2,6}+s_{\alpha}^{5,6}$, and thus $s_{\alpha}^{1,6} \leq s_{\alpha}^{2,6}+s_{\alpha}^{5,6}$. Furthermore, we have $\alpha\left(Q_{N}^{\prime}\right)=$ $b c a$, while Kemeny stability implies that for any $\dot{P}_{N} \in \Delta\left(Q_{N}^{\prime K}\right)$ that $S_{\alpha}^{6}\left(b c a, \dot{P}_{N}\right) \geq S_{\alpha}^{6}\left(b a c, \dot{P}_{N}\right)$. Hence, $(3 Z+1) s_{\alpha}^{1,6}+2 Z s_{\alpha}^{2,6}+2 Z s_{\alpha}^{5,6} \geq 2 Z s_{\alpha}^{1,6}+(3 Z+1) s_{\alpha}^{2,6}+3 Z s_{\alpha}^{5,6}$, leading to $s_{\alpha}^{1,6} \geq s_{\alpha}^{2,6}+\frac{Z}{Z+1} s_{\alpha}^{5,6}$ for all $Z>1$, Therefore, if $s_{\alpha}^{1,3}=2 s_{\alpha}^{2,3}$, then $s_{\alpha}^{1,6}=s_{\alpha}^{2,6}+s_{\alpha}^{5,6}$, and the proof is complete.

\section{A.2.3 Proof of Proposition 5}

Consider the following profiles $P_{N} \in \mathcal{L}\left(A_{3}\right)^{2 Z+2}$ and $P_{N}^{\prime} \in \mathcal{L}\left(A_{3}\right)^{56 Z+1}$, where $Z>1$ :

$$
P_{N}=\left(\begin{array}{ccc}
Z+1 & 1 & Z \\
\hline a & a & c \\
b & c & b \\
c & b & a
\end{array}\right) P_{N}^{\prime}=\left(\begin{array}{cccc}
11 Z & 28 Z & 17 Z & 1 \\
\hline a & a & b & c \\
b & c & c & b \\
c & b & a & a
\end{array}\right)
$$

Suppose that $s_{\alpha}^{1,3}<2 s_{\alpha}^{2,3}$. Then $\alpha\left(P_{N}\right)=b a c$ for $Z$ large enough. Moreover, from Proposition 3, $S_{\alpha}^{6}\left(\right.$ bac,$\left.\dot{P}_{N}\right)=(Z+1)\left(s_{\alpha}^{2,6}+s_{\alpha}^{5,6}\right)<S_{\alpha}^{6}\left(a c b, \dot{P}_{N}\right)=s_{\alpha}^{1,6}+(Z+1) s_{\alpha}^{2,6}+Z s_{\alpha}^{5,6}$ for all $\dot{P}_{N} \in \Delta\left(P_{N}^{K}\right)$, in contradiction with Kemeny-stability.

Suppose that $s_{\alpha}^{1,3}>2 s_{\alpha}^{2,3}$. Then $\alpha\left(P_{N}^{\prime}\right)=a b c$ for $Z$ large enough. Using again Proposition 3, $S_{\alpha}^{6}\left(a b c, \dot{P}_{N}\right)=11 Z s_{\alpha}^{1,6}+28 Z s_{\alpha}^{2,6}+17 Z s_{\alpha}^{5,6}$ while $S_{\alpha}^{6}\left(a c b, \dot{P}_{N}\right)=28 Z s_{\alpha}^{1,6}+11 Z s_{\alpha}^{2,6}+s_{\alpha}^{5,6}$ for all $\dot{P}_{N} \in$ $\Delta\left(P_{N}^{\prime K}\right)$. Since $s_{\alpha}^{5,6}>0$ from Proposition 3, we get by using Proposition $4, S_{\alpha}^{6}\left(a b c, \dot{P}_{N}\right)=39 Z s_{\alpha}^{2,6}+$ $28 Z s_{\alpha}^{5,6}<S_{\alpha}^{6}\left(a b c, \dot{P}_{N}\right)=39 Z s_{\alpha}^{2,6}+28 Z s_{\alpha}^{5,6}+s_{\alpha}^{5,6}$, in contradiction with Kemeny-stability.

\section{A.2.4 End of proof of Theorem 1}

(Necessary Part) Using Propositions 3,4 and 5, it suffices to prove that if $\alpha$ is Kemeny-stable, then $s_{\alpha}^{2,6}=3 s_{\alpha}^{5,6}$. Consider the following profiles $P_{N} \in \mathcal{L}\left(A_{3}\right)^{3 Z+1}$ and $P_{N}^{\prime} \in \mathcal{L}\left(A_{3}\right)^{3 Z-4}$, where $Z>2$ :

$$
P_{N}=\left(\begin{array}{cc}
2 Z+1 & Z \\
\hline a & b \\
b & c \\
c & a
\end{array}\right) P_{N}^{\prime}=\left(\begin{array}{ccc}
Z-1 & Z-1 & Z-2 \\
a & b & c \\
c & a & b \\
b & c & a
\end{array}\right)
$$

Suppose that $s_{\alpha}^{2,6}>3 s_{\alpha}^{5,6}$. Since $s_{\alpha}^{1,3}=2 s_{\alpha}^{2,3}$ from Proposition 5, then $\alpha\left(P_{N}\right)=a b c$. For any $\dot{P}_{N} \in \Delta\left(P_{N}^{K}\right)$, we get from Proposition 3 together with Proposition 4 that $S_{\alpha}^{6}\left(a b c, \dot{P}_{N}\right)=(2 Z+1) s_{\alpha}^{1,6}+$ $Z s_{\alpha}^{5,6}=(2 Z+1) s_{\alpha}^{2,6}+(3 Z+1) s_{\alpha}^{5,6}$, while $S_{\alpha}^{6}\left(b a c, \dot{P}_{N}\right)=(3 Z+1) s_{\alpha}^{2,6}$. But since $s_{\alpha}^{2,6}>3 s_{\alpha}^{5,6}$, we get $S_{\alpha}^{6}\left(b a c, \dot{P}_{N}\right)>S_{\alpha}^{6}\left(a b c, \dot{P}_{N}\right)$ for all $Z>2$, in contradiction with Kemeny-stability. 
Suppose that $s_{\alpha}^{2,6}<3 s_{\alpha}^{5,6}$. Using again $s_{\alpha}^{1,3}=2 s_{\alpha}^{2,3}$ from Proposition 5, we get $\alpha\left(P_{N}^{\prime}\right)=a b c$. For any $\dot{P}_{N} \in \Delta\left(P_{N}^{\prime K}\right)$, we get from Proposition 3 together with Proposition 4 that $S_{\alpha}^{6}\left(a b c, \dot{P}_{N}\right)=$ $(2 Z-2) s_{\alpha}^{2,6}$, while $S_{\alpha}^{6}\left(c b a, \dot{P}_{N}\right)=(Z-2) s_{\alpha}^{1,6}+(2 Z-2) s_{\alpha}^{5,6}=(Z-2) s_{\alpha}^{2,6}+(3 Z-4) s_{\alpha}^{5,6}$. Thus, $S_{\alpha}^{6}\left(b a c, \dot{P}_{N}\right)>S_{\alpha}^{6}\left(a b c, \dot{P}_{N}\right)$ for $Z$ large enough, in contradiction with Kemeny-stability. Hence one must have $s_{\alpha}^{2,6}=3 s_{\alpha}^{5,6}$, which proves the Necessary Part.

(Sufficiency Part). Consider any $n \in \mathbb{N}$ together with any profile $P_{N} \in \mathcal{L}\left(A_{3}\right)^{n}$ having the form

$$
P_{N}=\left(\begin{array}{cccccc}
n_{1} & n_{2} & n_{3} & n_{4} & n_{5} & n_{6} \\
\hline a & a & b & b & c & c \\
b & c & a & c & a & b \\
c & b & c & a & b & a
\end{array}\right)
$$

with $\sum_{h=1}^{6} n_{h}=n$. Pick up any scoring rule $\alpha$ fulfilling the conditions $(*) s_{\alpha}^{1,3}=2 s_{\alpha}^{2,3}>0$, and $(* *)$ $s_{\alpha}^{1,6}=\frac{4}{3} s_{\alpha}^{2,6}=\frac{4}{3} s_{\alpha}^{3,6}=4 s_{\alpha}^{4,6}=4 s_{\alpha}^{5,6}>s_{\alpha}^{6,6}=0$. We get that:

$-S_{\alpha}^{3}\left(a, P_{N}\right)=\left(2 n_{1}+2 n_{2}+n_{3}+n_{5}\right) s_{\alpha}^{2,3}$

$-S_{\alpha}^{3}\left(b, P_{N}\right)=\left(2 n_{3}+2 n_{4}+n_{1}+n_{6}\right) s_{\alpha}^{2,3}$

$-S_{\alpha}^{3}\left(c, P_{N}\right)=\left(2 n_{5}+2 n_{6}+n_{2}+n_{4}\right) s_{\alpha}^{2,3}$

Moreover, suppose without loss of generality that $s_{\alpha}^{5,6}=1$ and $a b c \in \Delta\left(\alpha\left(P_{N}\right)\right)$. It follows that:

$-n_{1}+2 n_{2}+n_{5} \geq n_{3}+2 n_{4}+n_{6}(1)$

$-2 n_{1}+n_{2}+n_{3} \geq n_{4}+n_{5}+2 n_{6}(2)$

$-n_{1}+2 n_{3}+n_{4} \geq n_{2}+2 n_{5}+n_{6}(3)$

Now, pick up any $\dot{P}_{N} \in \Delta\left(P_{N}^{K}\right)$. Then we get from $(* *)$ that:

$-S_{\alpha}^{6}\left(a b c, \dot{P}_{N}\right)=4 n_{1}+3\left(n_{2}+n_{3}\right)+\left(n_{4}+n_{5}\right)$

$-S_{\alpha}^{6}\left(a c b, \dot{P}_{N}\right)=4 n_{2}+3\left(n_{1}+n_{5}\right)+\left(n_{3}+n_{6}\right)$

- $S_{\alpha}^{6}\left(b a c, \dot{P}_{N}\right)=4 n_{3}+3\left(n_{1}+n_{4}\right)+\left(n_{2}+n_{6}\right)$

$-S_{\alpha}^{6}\left(b c a, \dot{P}_{N}\right)=4 n_{4}+3\left(n_{3}+n_{6}\right)+\left(n_{1}+n_{5}\right)$

$-S_{\alpha}^{6}\left(c a b, \dot{P}_{N}\right)=4 n_{5}+3\left(n_{2}+n_{6}\right)+\left(n_{1}+n_{4}\right)$

- $S_{\alpha}^{6}\left(c b a, \dot{P}_{N}\right)=4 n_{6}+3\left(n_{4}+n_{5}\right)+\left(n_{2}+n_{3}\right)$

Then one easily checks that $(3) \Rightarrow S_{\alpha}^{6}\left(a b c, \dot{P}_{N}\right) \geq S_{\alpha}^{6}\left(a c b, \dot{P}_{N}\right),(1) \Rightarrow S_{\alpha}^{6}\left(a b c, \dot{P}_{N}\right) \geq S_{\alpha}^{6}\left(b a c, \dot{P}_{N}\right)$, $(1)+(2) \Rightarrow S_{\alpha}^{6}\left(a b c, \dot{P}_{N}\right) \geq S_{\alpha}^{6}\left(b c a, \dot{P}_{N}\right),(2)+(3) \Rightarrow S_{\alpha}^{6}\left(a b c, \dot{P}_{N}\right) \geq S_{\alpha}^{6}\left(c a b, \dot{P}_{N}\right)$, and $(1)+(2)+(3) \Rightarrow S_{\alpha}^{6}\left(a b c, \dot{P}_{N}\right) \geq$ $S_{\alpha}^{6}\left(c b a, \dot{P}_{N}\right)$. Hence $a b c \in \Delta\left(\alpha\left(P_{N}\right)\right) \cap f_{\alpha}\left(\dot{P}_{N}\right)$, and the proof is complete.

\section{A.3 Proof of Theorem 2}

Let $\alpha$ be a non-truncated and Kemeny-stable scoring rule. Consider profile $P_{N} \in \mathcal{L}\left(A_{6}\right)^{A+B+C+1}$, where $A>B>C>1$ :

$$
P_{N}=\left(\begin{array}{llll}
A & B & C & 1 \\
\hline a & b & c & f \\
c & c & a & e \\
b & a & b & d \\
d & d & d & c \\
e & e & f & b \\
f & f & e & a
\end{array}\right)
$$


Using Theorem 1, and normalizing $S_{\alpha}^{6}$ by setting $s_{\alpha}^{1,6}=1$, we get $S_{\alpha}^{6}\left(a, P_{N}\right)=A+\frac{3}{4}(B+C)$, $S_{\alpha}^{6}\left(b, P_{N}\right)=\frac{3}{4}(A+C)+B+\frac{1}{4}, S_{\alpha}^{6}\left(c, P_{N}\right)=\frac{3}{4}(A+B)+C+\frac{1}{4}, S_{\alpha}^{6}\left(d, P_{N}\right)=\frac{1}{4}(A+B+C)+\frac{3}{4}$, $S_{\alpha}^{6}\left(e, P_{N}\right)=\frac{1}{4}(A+B)+\frac{3}{4}$, and $S_{\alpha}^{6}\left(f, P_{N}\right)=\frac{1}{4} C+1$. Obviously, $A, B$ and $C$ can be chosen to ensure that $\alpha\left(P_{N}\right)=a b c d e f$. Consider the following Kemeny hyper-profile $\dot{P}_{N} \in \Delta\left(P_{N}^{K}\right)$

$$
\dot{P}_{N}=\left(\begin{array}{cccc}
A & B & C & 1 \\
\hline \text { acbdef } & \text { bcadef } & \text { cabdfe } & \text { fedcba } \\
\text { cabdef } & \text { cbadef } & \text { cabdef } & \ldots \\
\text { abcdef } & \text { bacdef } & \text { cbadfe } & \ldots \\
\ldots & \text { bcdaef } & \text { cadbfe } & \ldots \\
\ldots & \text { bcaedf } & \text { cabfde } & \ldots \\
\ldots & \text { bcadfe } & \text { acbdfe } & \ldots \\
\ldots & \text { cabdef } & \ldots & \ldots \\
\ldots & \text { abcdef } & \ldots & \ldots \\
\ldots & \ldots & \ldots & \ldots
\end{array}\right)
$$

We get that $S_{\alpha}^{6 !}\left(\right.$ cabdef,$\left.\dot{P}_{N}\right)=(A+C) s_{\alpha}^{2,6 !}+B s_{\alpha}^{7,6 !}+s_{\alpha}^{z, 6 !}$, where $z<6 !$, whereas $S_{\alpha}^{6 !}\left(\right.$ abcdef,$\left.\dot{P}_{N}\right)=$ $A s_{\alpha}^{3,6 !}+B s_{\alpha}^{8,6 !}+C s_{\alpha}^{w, 6 !}$, where $w>6$. Finally, Kemeny-stability implies that $s_{\alpha}^{1,6 !}=\ldots=s_{\alpha}^{8,6 !}$, and $s_{\alpha}^{z, 6 !}=0$, which contradicts that $\alpha$ is non-truncated.

\section{A.4 Proof of Theorem 3}

The proof is similar to the one above. Consider profile $P_{N} \in \mathcal{L}\left(A_{6}\right)^{9}$ below:

$$
P_{N}=\left(\begin{array}{llll}
4 & 3 & 1 & 1 \\
\hline a & b & c & c \\
c & c & a & b \\
b & a & b & a \\
d & d & d & d \\
e & e & e & e \\
f & f & f & f
\end{array}\right)
$$

We get $S_{\alpha}^{6}\left(a, P_{N}\right)=4 s_{\alpha}^{1,6}+s_{\alpha}^{2,6}+4 s_{\alpha}^{3,6}, S_{\alpha}^{6}\left(b, P_{N}\right)=3 s_{\alpha}^{1,6}+s_{\alpha}^{2,6}+5 s_{\alpha}^{3,6}, S_{\alpha}^{6}\left(c, P_{N}\right)=2 s_{\alpha}^{1,6}+7 s_{\alpha}^{2,6}$, $S_{\alpha}^{6}\left(d, P_{N}\right)=9 s_{\alpha}^{4,6}, S_{\alpha}^{6}\left(e, P_{N}\right)=9 s_{\alpha}^{5,6}$, and $S_{\alpha}^{6}\left(f, P_{N}\right)=0$. If $\alpha$ is Kemeny-stable, it follows from Theorem 1 that $s_{\alpha}^{2,6}=s_{\alpha}^{3,6}$, which implies that $\Delta\left(\alpha\left(P_{N}\right)\right) \subseteq\left\{P \in \mathcal{L}\left(A_{6}\right): P=(a b c \rightarrow Q)\right.$, where $Q \in$ $\mathcal{L}(\{d, e, f\})\}$. Consider the following Kemeny hyper-profile $\dot{P}_{N} \in \Delta\left(P_{N}^{K}\right)$ 


$$
\dot{P}_{N}=\left(\begin{array}{cccc}
4 & 3 & 1 & 1 \\
\hline \text { acbdef } & \text { bcadef } & \text { cabdef } & \text { cbadef } \\
\text { cabdef } & \text { cbadef } & \text { acbdef } & \text { cabdef } \\
\text { abcdef } & \text { bacdef } & \text { cbadef } & \text { bcadef } \\
\ldots & \text { bcdaef } & \text { cadbef } & \text { cbdaef } \\
\ldots & \text { bcaedf } & \text { cabedf } & \text { cbaedf } \\
\ldots & \text { bcadfe } & \text { cabdfe } & \text { cbadfe } \\
\ldots & \text { cabdef } & \text { abcdef } & \text { abcdef } \\
\ldots & \text { abcdef } & \ldots & \ldots \\
\ldots & \ldots & \ldots & \ldots
\end{array}\right)
$$

We get that $S_{\alpha}^{6 !}\left(\right.$ cabdef,$\left.\dot{P}_{N}\right)=4 s_{\alpha}^{2,6 !}+3 s_{\alpha}^{7,6 !}+s_{\alpha}^{1,6 !}+s_{\alpha}^{2,6 !}$, whereas $S_{\alpha}^{6 !}\left(\operatorname{abcdef}, \dot{P}_{N}\right)=4 s_{\alpha}^{3,6 !}+$ $3 s_{\alpha}^{8,6 !}+s_{\alpha}^{7,6 !}+s_{\alpha}^{7,6 !}$. Using $s_{\alpha}^{2,6 !} \geq s_{\alpha}^{3,6 !}$ and $s_{\alpha}^{7,6 !} \geq s_{\alpha}^{8,6 !}$ together with the strict-at-top property, we have that $S_{\alpha}^{6 !}\left(\right.$ cabdef,$\left.\dot{P}_{N}\right)>S_{\alpha}^{6 !}\left(\right.$ abcdef,$\left.\dot{P}_{N}\right)$. The conclusion follows from the fact that abcdef maximizes $S_{\alpha}^{6 !}\left(P, \dot{P}_{N}\right)$ in $\Delta\left(\alpha\left(P_{N}\right)\right)$.

\section{A.5 Proof of Theorem 5}

Consider profile $P_{N} \in \mathcal{L}\left(A_{3}\right)^{5}$ below:

$$
P_{N}=\left(\begin{array}{cccc}
2 & 1 & 1 & 1 \\
\hline a & b & c & c \\
b & a & b & a \\
c & c & a & a
\end{array}\right)
$$

Pick up a scoring rule $\alpha$ hyper-stable for $\mathcal{S}$. Since $\mathcal{K} \subset \mathcal{S}$, then $\alpha$ is Kemeny-stable. It follows from Theorem 1 that score vectors must be such that $(*) s_{\alpha}^{1,3}=2 s_{\alpha}^{2,3}>0$, and $(* *) s_{\alpha}^{1,6}=\frac{4}{3} s_{\alpha}^{2,6}=\frac{4}{3} s_{\alpha}^{3,6}=$ $4 s_{\alpha}^{4,6}=4 s_{\alpha}^{5,6}>s_{\alpha}^{6,6}=0$. It follows that $\alpha\left(P_{N}\right)=a b c$. It is straightforward to check that the following hyper-profile $P_{N}^{E}$ is built from a 5-tuple $E=\left(e_{1}, e_{1}, e_{3}, e_{4}, e_{5}\right)$ of separable preference extensions:

$$
P_{N}^{E}=\left(\begin{array}{cccc}
2 & 1 & 1 & 1 \\
\hline a b c & b a c & c b a & c a b \\
b a c & b c a & b c a & a c b \\
a c b & a b c & b a c & c b a \\
b c a & a c b & c a b & b c a \\
c a b & c b a & a c b & a b c \\
c b a & c a b & a b c & b a c
\end{array}\right)
$$

Note that all extensions in $E$ but $e_{4}$ are Kemeny. We get $S_{\alpha}^{6}\left(a b c, P_{N}^{E}\right)=12 s_{\alpha}^{5,6}<S_{\alpha}^{6}\left(b a c, P_{N}^{E}\right)=$ $13 s_{\alpha}^{5,6}$, which contradicts hyper-stability for $\mathcal{S}$. 


\section{A.6 Proof of Theorem 6}

Given any $Q \in \mathcal{L}\left(A_{m}\right)$, we write $Q=\left(Q_{1} \rightarrow Q_{2} \rightarrow \ldots \rightarrow Q_{H}\right)$, where, for any $1 \leq h \leq H$, $Q_{h} \in \mathcal{L}\left(B_{h}\right)$ is a segment of $Q$, and where $\left\{B_{1}, B_{2}, \ldots, B_{H}\right\}$ is a partition of $A_{m}$ into non-empty sets. Lemma 7 is a useful intermediate step towards the proof.

Lemma 7 Let $Q, Q^{\prime} \in \mathcal{L}\left(A_{m}\right)$ be respectively defined by $Q=\left(Q_{1} \rightarrow x \rightarrow Q_{2} \rightarrow y \rightarrow Q_{3}\right)$ and $Q^{\prime}=$ $\left(Q_{1} \rightarrow y \rightarrow Q_{2} \rightarrow x \rightarrow Q_{3}\right)$, where $Q_{h} \in \mathcal{L}\left(B_{h}\right)$, for $1 \leq h \leq 3$. Then, for any $P_{N} \in \mathcal{L}\left(A_{m}\right)^{n}$ with $n$ is odd, and any $E=\left(e_{1}, \ldots, e_{n}\right) \in \mathcal{S}^{n},\left[x \mu\left(P_{N}\right) y\right] \Rightarrow\left[Q \mu\left(P_{N}^{E}\right) Q^{\prime}\right]$,

Proof: Define $B=\{x, y\} \cup B_{2}$, where $Q_{2} \in \mathcal{L}\left(B_{2}\right)$. Pick up any $P_{i} \in \mathcal{L}\left(A_{m}\right)$ where $x P_{i} y$, and consider the restriction $\left.P_{i}\right|_{B}$ of $P_{i}$ to $B$. We can write $\left.P_{i}\right|_{B}=\left(V_{1} \rightarrow x \rightarrow V_{2} \rightarrow y \rightarrow V_{3}\right)$, where $V_{1}$, $V_{2}$, and $V_{3}$ are segments of $\left.P_{i}\right|_{B}$, with $V_{h} \in \mathcal{L}\left(B_{2 h}\right), 1 \leq h \leq 3$, and $\left\{B_{21}, B_{22}, B_{23}\right\}$ being a partition of $B_{2}$. Then $A\left(\left.P_{i}\right|_{B},\left.Q\right|_{B}\right)=\{x, y\} \cup\left[\{x\} \times\left(B_{22} \cup B_{23}\right)\right] \cup\left[\left(B_{21} \cup B_{22}\right) \times\{y\}\right] \cup A\left(\left.P_{i}\right|_{B_{2}}, Q_{2}\right)$, while $A\left(\left.P_{i}\right|_{B},\left.Q^{\prime}\right|_{B}\right)=A\left(\left.P_{i}\right|_{B},\left.Q\right|_{B}\right) /\{x, y\}$. Hence, $A\left(\left.P_{i}\right|_{B},\left.Q^{\prime}\right|_{B}\right) \subset A\left(\left.P_{i}\right|_{B},\left.Q\right|_{B}\right)$. Since $Q$ and $Q^{\prime}$ have the same segment $Q_{1}$ at top and the same segment $Q_{3}$ at bottom, then $A\left(P_{i}, Q^{\prime}\right) \subset A\left(P_{i}, Q\right)$. From separability of $e_{i}$, we get $Q e_{i}\left(P_{i}\right) Q^{\prime}$. Finally, $x \mu\left(P_{N}\right)$ y implies that $\left|\left\{i: x P_{i} y\right\}\right|>\frac{n}{2}$, hence that $\left|\left\{i: Q e_{i}\left(P_{i}\right) Q^{\prime}\right\}\right|>\frac{n}{2}$ and the conclusion follows.

Given $P_{N} \in \mathcal{L}\left(A_{m}\right)^{n}$, the top-cycle for $P_{N}$ is the subset $T\left(B, P_{N}\right)$ of $A_{m}$ containing all maximal elements for $\theta\left(P_{N}\right)$. The transitive closure partition of $A_{m}$ is the ordered set $S\left(\theta, P_{N}\right)=\left(S_{1}, S_{2}, \ldots, S_{J}\right)$ of indifference classes for $\theta\left(P_{N}\right)$, where $\forall j \leq j^{\prime} \in\{1, \ldots, J\}, \forall\left(x, x^{\prime}\right) \in S_{j} \times S_{j^{\prime}}, x \theta\left(P_{N}\right) x^{\prime}$ and $\neg\left(x^{\prime} \theta\left(P_{N}\right) x\right.$ if $j<j^{\prime}$. By definition of $\theta$, one has $\Delta\left(\theta\left(P_{N}\right)\right)=\left\{Q \in \mathcal{L}\left(A_{m}\right): Q=\left(Q_{1} \rightarrow Q_{2} \rightarrow \ldots \rightarrow Q_{J}\right)\right.$ where, for each $\left.j=1, \ldots, J, Q_{j} \in \mathcal{L}\left(S_{j}\right)\right\}$. The proof of Theorem 6 is complete if we show that for any $E=\left(e_{1}, \ldots, e_{n}\right) \in \mathcal{S}^{n}, \Delta\left(\theta\left(P_{N}\right)\right) \cap T\left(\mathcal{L}\left(A_{m}\right), P_{N}^{E}\right) \neq \varnothing$.

Pick up any $P \in \mathcal{L}\left(A_{m}\right) / \Delta\left(\theta\left(P_{N}\right)\right)$ and any $E=\left(e_{1}, \ldots, e_{n}\right) \in \mathcal{S}^{n}$. Define $B(P)=\left\{x \in A_{m}: x \in S_{j}\right.$ for some $j$ and $\left.\forall y \in S_{j^{\prime}} /\{x\}, x P y \Rightarrow j^{\prime}>j\right\}$, and $B=A_{m} / B(P)$. Consider order $Q(P) \in \Delta\left(\theta\left(P_{N}\right)\right)$ such that:

- $\left.Q(P)\right|_{B(P)}=\left.P\right|_{B(P)}$

- $x P y \Rightarrow x Q(P) y$ for all $x, y \in B \cap S_{j}$ for some $j \in\{1, \ldots, J\}$.

Write $\left.P\right|_{B}=b_{1} b_{2} \ldots b_{T}$, where $T=|B|$. There exists a permutation $\sigma$ of $\{1, \ldots, T\}$ such that $\left.Q(P)\right|_{B}=$ $b_{\sigma(1)} b_{\sigma(2)} \cdots b_{\sigma(T)}$. Then, there is a finite sequence $\left\{\omega_{h}\right\}_{1 \leq h \leq H}$ of transpositions of $A_{m}$, where $H \leq T$, such that $\omega_{1}$ swaps $b_{1}$ and $b_{\sigma(1)}$ in $\left.P\right|_{B}$, leading to $\left.P^{1}\right|_{B}=b_{\omega_{1}(1)} b_{\omega_{1}(2)} \ldots b_{\omega_{1}(T)}, \omega_{2}$ swaps $b_{\omega_{1}(2)}$ and $b_{\sigma(2)}$ in $\left.P^{1}\right|_{B}$, leading to $\left.P^{2}\right|_{B}=b_{\omega_{2} \circ \omega_{1}(1)} b_{\omega_{2} \circ \omega_{1}(2)} \ldots b_{\omega_{2} \circ \omega_{1}(T)}, \ldots, \omega_{H}$ swaps $b_{\sigma(T)}$ and $b_{\omega_{T-1} \circ \ldots \circ \omega_{1}(T)}$ in $\left.P^{T-1}\right|_{B}$, leading to $\left.P^{T}\right|_{B}=\left.Q(P)\right|_{B}$. Since $b_{\sigma(1)} \mu\left(P_{N}\right) b_{\sigma(2)} \mu\left(P_{N}\right) \ldots \mu\left(P_{N}\right) b_{\sigma(T)}$, then Lemma 7 ensures that for all $1 \leq h \leq H$, either $\left.P^{h+1}\right|_{B}=\left.P^{h}\right|_{B}$ or $\left(\left.P^{h+1}\right|_{B}\right) \mu\left(\left.P_{N}^{E}\right|_{B}\right)\left(\left.P^{h}\right|_{B}\right)$. Hence $\left(\left.Q(P)\right|_{B}\right.$ $\theta\left(\left.P_{N}^{E}\right|_{B}\right)\left(\left.P\right|_{B}\right)$, and thus $Q(P) \theta\left(P_{N}^{E}\right) P$. This proves that for any order $P$ not in $\Delta\left(\theta\left(P_{N}\right)\right)$, there exists $Q \in \Delta\left(\theta\left(P_{N}\right)\right)$ such that $Q \theta\left(P_{N}^{E}\right) P$.

Finally, since $T\left(\mathcal{L}\left(A_{m}\right),\left.P_{N}^{E}\right|_{\Delta\left(\theta\left(P_{N}\right)\right)}\right) \neq \varnothing$, there exists $Q \in \Delta\left(\theta\left(P_{N}\right)\right)$ such that $Q \theta\left(P_{N}^{E}\right) Q^{\prime}$ for all $Q^{\prime} \in \Delta\left(\theta\left(P_{N}\right)\right) /\{Q\}$. Thus, there exists $Q \in \Delta\left(\theta\left(P_{N}\right)\right)$ such that $Q \theta\left(P_{N}^{E}\right) Q^{\prime}$ for all $Q^{\prime} \in \mathcal{L}\left(A_{m}\right) /\{Q\}$. Thus $\left.\Delta\left(\theta\left(P_{N}\right)\right) \cap T\left(\mathcal{L}\left(A_{m}\right), P_{N}^{E}\right)\right) \neq \varnothing$ and the proof is complete. 


\section{A.7 Proof of Proposition 2}

Let $P_{N}=\left(P_{1} \ldots, P_{i}, \ldots, P_{n}\right) \in \mathcal{L}\left(A_{m}\right)^{n}$. Choose any $E=\left(e_{1}, \ldots, e_{n}\right) \in \mathcal{S}^{n}$. Suppose without loss of generality that $\mu\left(P_{N}\right)=a_{1} a_{2} \ldots a_{m}$. Moreover, suppose that $C W\left(P_{N}^{E}\right)=Q=b_{1} b_{2} \ldots b_{m}$, with $b_{1} \neq a_{1}=$ $b_{h}$ for some $2 \leq h \leq m$. Now define $Q^{\prime}=a_{1} b_{2} \ldots b_{h-1} b_{h} b_{h+1} \ldots b_{m} \in \mathcal{L}\left(A_{m}\right)$. It follows from Lemma 7 above that $\left[1 \mu\left(P_{N}\right) b_{1}\right] \Rightarrow\left[Q^{\prime} \mu\left(P_{N}^{E}\right) Q^{\prime}\right]$, which contradicts $C W\left(P_{N}^{E}\right)=Q$. Thus, $b_{1}=a_{1}$. We conclude by iterating the same argument for $b_{2}, \ldots, b_{m}$. 